Check for updates

Cite this: RSC Adv., 2017, 7, 35060

Received 22nd June 2017

Accepted 6th July 2017

DOI: $10.1039 / c 7 r a 06981 b$

rsc.li/rsc-advances

\section{Carbon allotropes grafted with poly(pyrrole) derivatives via living radical polymerizations: electrochemical analysis of nano-composites for energy storage $\dagger$}

\begin{abstract}
Mariusz Radtke and Anna Ignaszak (iD *
Carbon-based nanomaterials are key components in energy storage devices. Their functions can be tailored by adjusting or developing new synthesis pathways. In this study, two living radical polymerization techniques, an electrochemically-aided atom transfer radical polymerization (e-ATRP) and reversible addition chain transfer polymerization (RAFT), were applied for grafting of carbon allotropes such as multi-walled carbon nanotubes (MWCNT), graphene and single-walled carbon nanohorns (SWCNH) with a 2-(1H-pyrrol-1-yl)ethyl methacrylate. The functionalized carbons were examined as polymerization initiators in the RAFT and e-ATRP synthesis. The FTIR and Raman spectroscopies were used to identify the reaction products at each step of preparation and for final composites. TEM imaging demonstrated that the morphology of composites made via RAFT and e-ATRP using the same carbon were not significantly different. The electrochemical analysis of grafted materials showed an improved gravimetric capacitance as compared to their individual components, revealing the synergy of a double-layer and pseudo-capacitance.
\end{abstract}

\section{Introduction}

As the world needs more energy than ever before, implementation of clean energy technologies in forms of energy storage (batteries, capacitors) and energy conversion (fuel cells) in everyday lives becomes a necessity that must be addressed promptly. The number of users of portable electronics is rapidly increasing and utilization of energy storage components will grow accordingly. In the perspective of the next few decades, several issues have to be solved with regards to current technologies. One of the most serious is disposal of batteries/ capacitors made from heavy or toxic metals (cadmium, lead, lithium). Another bottleneck is limited access to resources of ores and metals oxides used for cathodes. ${ }^{1}$ The necessity for replacement (or decrease in usage) of conventional materials used in batteries, ultra- and supercapacitors, is pushing research towards completely new alternatives. ${ }^{2}$ This task is not

Department of Chemistry, University of New Brunswick, 30 Dineen Drive, Fredericton, NB, E3B 5A3, Canada. E-mail: Anna.Ignaszak@unb.ca; Tel: +1 5064477107

$\dagger$ Electronic supplementary information (ESI) available: NMR spectra of RAFT and e-ATRP reagents, Raman and FTIR spectra of RAFT and e-ATRP reagents before polymerization, electrochemical setup for synthesis of e-ATRP initiators, Tafel plots of e-ATRP initiators, EDS spectra of final products, and TEM images of pristine carbon allotropes. The Table S1 demonstrates gravimetric capacitance of pristine carbons and a pure PPy used as reference samples. The Fig. S16 shows results of electro-polymerization of the pyrrole (carried out in order to compare the electrochemical performance of this materials with the product synthesized using a chemical crosslinking in the presence of APS, used in this study). See DOI: 10.1039/c7ra06981b easy - new electrode materials should fulfill many requirements, such as a long-life cycle stability, high specific gravimetric capacitance, and fast energy uptake and release. ${ }^{3,4}$ In order to overcome above challenges, these materials should be designed on a molecular level, resulting in a control/improvement of their morphological, structural and electrochemical characteristics.

A strong position in the area of metal-free electrodes is given to nanostructured carbons, especially multi-walled carbon nanotubes (MWCNTs) and graphene, due to their remarkable high electrochemical double layer capacitance (EDLC). ${ }^{5}$ These carbons can be paired with other capacitive materials, such as redox-active polymers. In capacitor field, this conjugation results in synergistic improvement of a total electrode capacitance, ${ }^{6}$ and more importantly, such composites usually outperform their metallic peers in terms of electrochemical and mechanical stability. ${ }^{7,8}$ The carbons grafted with particular conducting polymers or two-dimensional oxides also show higher energy density than conventional electrochemical double layer capacitors (EDLCs). ${ }^{4,9}$ Suitable carbons for grafting are MWCNTs, graphene, and relatively new, singlewalled carbon nanohorns (SWCNHs), due to primarily high specific surface area and tailored pore size that facilitate fast adsorption/desorption of ions from operating electrolyte. This enables an ultra-fast recovery of the double-layer capacitance, leading to the charge-discharge time counted in milliseconds. ${ }^{10}$

The pseudo-capacitive function of conjugated polymers is well-known process and relies on the reduction-oxidation of the polymer chain, and the corresponding doping of counter ions from the electrolyte. ${ }^{11}$ These reversible redox reactions are 
foundation for the fast charge-discharge processes. ${ }^{12}$ The combined EDLC and pseudocapacitive materials are called hybrid capacitors. Usually, the carbon-polymer hybrids used in supercapacitors perform better than their individual components, ${ }^{13}$ however they suffer from poor electrochemical stability. ${ }^{\mathbf{1 4}}$ The improvement of capacitance in such hybrids is generated from strong electronic interactions between the carbon graphitized surface and the $\pi$ electrons of conjugated double-bonds in polymer chain, resulting in areas with enriched electron density, and thus stronger attraction of a counterion from the electrolyte. ${ }^{15}$ However, considerable loss of pseudocapacitance caused by the volumetric changes in the polymer upon cycling and its oxidation, lead to the short cycle stability of these combined capacitor electrodes. ${ }^{16}$

The stability is significantly improved via a grafting of carbon surface with the polymer through appropriate covalent bond, resulting in new, highly ordered molecular structures. ${ }^{\mathbf{1 3 , 1 4}}$ A rigid and electrochemical stable covalent linker, connecting the double-layer (carbon) and pseudo-capacitor (polymer) is vital to the success of composite electrodes. Such molecular bond provides the necessary electronic linkage between the carbon and polymer. Its function is even more important since it controls an extent of distribution and the coverage of polymer onto the carbon surface. The electrochemical function of the molecular linker is critical in overall performance of the composite electrode: (1) it should facilitate an ion diffusion to the redox active centers of the polymer without obstructing the double-layer interface (carbon); and (2) it should contribute to (or at least not hamper) good electron conductivity and associated electronic effects, leading to the improved chemical, mechanical and electrochemical stability. ${ }^{\mathbf{4 , 1 4}}$ The concentration and distribution of a molecular bridge dictates the morphology of the polymer coating, ${ }^{17}$ and therefore plays critical role in sustaining electronic effects, leading to desired electrode response and the stability.

A number of successful carbon grafting methods with conjugated polymers via rigid chemical anchorage rely on chemical pathways (with a 3 -aminobenzoic acid linker, ${ }^{14}$ amide derivative ${ }^{\mathbf{1 6}}$ ), or electrochemical functionalization, such as in situ reduction of diazonium salts on various carbon electrodes. ${ }^{18}$ These synthesis methods begin from functionalization of carbon in the first step, and the attachment of the conjugated polymer to molecular linker (bridge with carbon) in last phase of preparation. ${ }^{\mathbf{1 4 , 1 6}}$ Although, these methods deliver composites with high gravimetric capacitance and a long-term life cycle, there is still room for improvement, as performance of carbon based polymeric composites strongly depends on the overall morphology in molecular and macroscopic scale.

A control over the polymer structure can be achieved via living radical polymerizations. ${ }^{19}$

The living radical polymerizations allow to control the rate of polymerization, and thus to generate more homogenous nanostructures. The propagation of polymer chains of same length causes the ratio of an average molecular weight and the number of average molecular weights to reach unity. ${ }^{20}$ This means that the predicted molecular weight of the macromolecule will be evenly distributed within whole polymer particle. This feature can be analyzed by the size exclusion chromatography (SEC) or the matrix assisted laser desorption ionization time of flight mass spectrometry (MALDI TOF MS). However, the SEC technique requires the polymer to be soluble. In case of nanocomposites utilized in this study, both the poly(pyrrole) and carbon allotrope are insoluble. This makes the estimation of PDI index and other characterizations of our composites very problematic. $^{21}$ In general, techniques such as a Reversible Addition Fragmentation Chain Transfer (RAFT) and the electrochemically aided Atom Transfer Radical Polymerization (eATRP) allow to control better the uniformity of polymeric chain as compared to the conventional chemical polymerizations (due to the significantly improved polydispersity index, leading to values close to the unity). ${ }^{20}$ Another important benefit of these methods, especially when carbon allotropes are used as polymerization initiators, is quenching/inhibiting of monomer homopolymerization. ${ }^{22}$ This is particularly important for the carbon-grafted polymer composites, since the particle size of both functional components is in the range of nanometers.

In this study, the modified carbon allotropes bearing respective dithioester or alkyl halides active groups are applied as initiators for the living radical polymerizations of a methacrylate-functionalized pyrrole monomer. The nanostructured carbons (source of double-layer capacitance) such as the MWCNTs, graphene and relatively new carbon allotrope that is single-walled carbon nanohorns (SWCNHs), are used for the first time as initiators during the living radical polymerization. These carbons are grafted via the e-ATRP and RAFT techniques with a 2-(1H-pyrrol-1-yl) ethyl methacrylate monomer in order to attach a polymer (pseudo-capacitor). The material characterization using microscopic and spectroscopic methods is correlated with the electrochemical activity for all newly developed composites. In the proposed synthetic methodology, the derivatization of polymer with the molecular linker is carried out first, and its polymerization onto the carbon takes place after, which is in reverse order as compared to the synthesis methods reported in literature. Since the structure and the particle size of the single-walled carbon nanohorns are very different from other two carbons used in this work (MWCNTs and graphene), its function as the e-ATRP and RAFT polymerization initiator provide new important insight regarding its processability and possible applications in electrochemical energy storage.

\section{Experimental section}

\subsection{Materials and methods}

Unless otherwise stated all materials were used as received without further purification. All solvents were obtained in their anhydrous from department-installed solvent purification system. A 2-propanol (for molecular biology, BioReagent, C99.5\%, I9516 SigmaAldrich), single-walled carbon nanohorns (as-grown, 804118 Sigma-Aldrich, BET $400 \mathrm{~m}^{2} \mathrm{~g}^{-1}$ ), DCM (ACS reagent, C99.5\%, containing 50 ppm amylene as a stabilizer, 443484 Sigma-Aldrich), dimethylformamide (anhydrous, 99.8\%, 227056 Sigma-Aldrich), multi-walled carbon nanotubes (O.D. 9 L 6-9 nm, 95\% (carbon), 724769 Sigma-Aldrich, BET $220 \mathrm{~m}^{2} \mathrm{~g}^{-1}$ ), poly(vinylidene difluoride) with an average $M_{\mathrm{w}}=180.000$, potassium bromide (FTIR 
grade), potassium chloride (analytical grade, p.a., C99.5\%), THF (anhydrous, C99.9\%, inhibitor-free), 1-(2-bromoethyl)-1H-pyrrole (98\%), 2-bromoisobutyryl bromide (99\%), chloroethylmagnesium (99\%), carbon disulfide (>99\%), copper(I) bromide, $p$-toluenesulfonic acid, sodium nitrite; a 2,2'-azobis(2-methylpropionitrile) (AIBN, purity > 99\%), and a 4-dimethylaminopyridine (DMAP) were purchased from Sigma-Aldrich Canada. Single-layer graphene flakes (grade AO-3, $12 \mathrm{~nm}$ flakes, specific surface area $80 \mathrm{~m}^{2} \mathrm{~g}^{-1}$, and purity 99.2\%) were obtained from Calverton, NY, USA. A 3aminobenzoic acid (Sigma-Aldrich Canada) was recrystallized three times from absolute ethanol prior use. PMDETA (SigmaAldrich Canada) was deoxygenated and stored under inert atmosphere, hydrochloric acid (37\%) was supplied by VWR Canada, and a triethylamine (Sigma Aldrich) was distilled under inert atmosphere and stored in a Schlenk flask over 4 A molecular sieves. The carbon black (Super P conductive, 99\%, metal basis) was purchased from Alfa Aesar, UK. TEM imaging was carried out using a Jeol 2100 sTEM operating at $200 \mathrm{kV}$. FTIR spectra on a $\mathrm{KBr}$ pellet were recorded with the NicoletTM iSTM 50 FTIR, $4 \mathrm{~cm}^{-1}$ resolution, 100 scan per sample ( $\mathrm{KBr}$ used for the pellets was dried in oven at $80{ }^{\circ} \mathrm{C}$ prior the use). The Raman spectra were recorded with a Renishaw inVia Raman spectrometer at $785 \mathrm{~nm}$ excitation source (Renishaw Inc, UK) in spectral range from 1000 to $3000 \mathrm{~cm}^{-1}$ and $0.1 \mathrm{~mW}$ laser power using 50 times magnification on the microscope. All electrochemical experiments were carried out using a $\mathrm{CH}$ Instruments electrochemical workstation model C760 and with an electrochemical cell containing a platinum wire counter electrode (CE), Ag/AgCl (1 M KCl $0.222 \mathrm{~V}$ ) or non-aqueous Ag/ $\mathrm{Ag}^{+}$reference electrode (RE), and the ink-deposited glassy carbon rotating disk electrode (RDE) as a working electrode (WE). The working electrode was mounted to the Pine AFMSRCE rotating disk electrode station. All potentials were quoted $v s$. $\mathrm{Ag} / \mathrm{AgCl}(222 \mathrm{mV}$ vs. standard hydrogen electrode) and all electrochemical measurements were carried out in $1 \mathrm{M} \mathrm{KCl}$ purged with $\mathrm{N}_{2}$ for 30 min prior to experiments $\left(\mathrm{N}_{2}\right.$ gas blanket was kept during the measurement). The glassy carbon working electrode disk of $0.5 \mathrm{~cm}$ diameter (PINE Instrument Company, USA) was mechanically polished with $0.05 \mu \mathrm{m} \mathrm{Al}{ }_{2} \mathrm{O}_{3}$ slurry (Cypress Systems Inc., USA), rinsed in double-distilled water, sonicated for $5 \mathrm{~min}$, finally rinsed with isopropanol and ethanol and dried under a gentle stream of air. Ink was prepared by dissolving PVDF ( $2 \mathrm{mg}$ ) in isopropanol $(2 \mathrm{~mL})$, sonicated for $0.5 \mathrm{~h}$ together with carbon black ( $2 \mathrm{mg}$ ) and $8.5 \mathrm{mg}$ of an active material. The resulting suspension was dropped $(17 \mu \mathrm{L}$ overall with $8.5 \mu \mathrm{L}$ for each casting portion) onto the polished glassy carbon working electrode $\left(0.1963 \mathrm{~cm}^{2}\right.$ area $)$ and dried for 2 min under a $60 \mathrm{~W}$ lamp. The cyclic voltammetry (CV) scans were conducted in the potential range from 0.8 to $-0.8 \mathrm{~V}$ ( $v s$. $\mathrm{Ag} / \mathrm{AgCl} 0.222 \mathrm{~V}$ ), chronocoulometry was examined at $0.3 \mathrm{~V}$ that is an onset potential of the polypyrrole oxidation. The electrochemical stability test was carried out using chronopotentiometry (CP) technique by sweeping the potential at various anodic and cathodic current loads until a clear destruction of the electrode was observed (as indicated in the ESI, Fig. S14†).

\subsection{Synthesis}

Fig. 1 represents the consecutive steps in the synthesis of MWCNTs/graphene/SWCNHs-based initiators (a and b) and the 2-(1-H-pyrrol-1-yl)ethyl methacrylate monomer (c). Carbon allotropes grafted with a $p$-aminoaniline were prepared according to the procedure described previously. ${ }^{16}$

2.2.1. Synthesis of the 2 -( $1 \mathrm{H}$-pyrrol-1-yl)ethyl methacrylate (monomer). $1 \mathrm{~g}$ of methacrylic acid $\left(\rho=1.015 \mathrm{~g} \mathrm{~cm}^{-3}\right)$ was purified under the glove bag filled with dry nitrogen by a passage through a short silica column in order to remove hydroquinone monomethyl ether stabilizer. Further, $0.246 \mathrm{~g}$ of methacrylic acid was introduced to a $10 \mathrm{~mL}$ Schlenk flask and mixed with $5 \mathrm{~mL}$ of dry $\mathrm{CH}_{3} \mathrm{CN}, 0.25 \mathrm{~mL}$ of 2-bromoethyl-1- $\mathrm{H}$ pyrrole and 2-fold excess of triethylamine (distilled under dry nitrogen and stored under $4 \AA$ molecular sieves prior use). The flask was closed with a reflux condenser attached to a balloon filled with dry dinitrogen. The apparatus was removed from the glove-bag, the cooling hoses were connected to the condenser and the flask was immersed in the pre-heated at $82{ }^{\circ} \mathrm{C}$ silicon oil bath for $15 \mathrm{~h}$. After the reaction was completed, $\mathrm{CH}_{3} \mathrm{CN}$ was evaporated under reduced pressure and the crude was redissolved in $10 \mathrm{~mL}$ chloroform (wet). The solution was washed five times with $10 \mathrm{~mL}$ in of a concentrated sodium hydrogen sulfate solution and brine in a separatory funnel (to remove protonated triethylamine bromide salt and potentially unreacted methacrylic acid), dried over magnesium sulfate, filtered under air pressure and evaporated under reduced pressure (colorless oil). The ${ }^{1} \mathrm{H}$ NMR $\left(300 \mathrm{MHz}, \mathrm{CDCl}_{3}, \delta\right): 6.7$ (t, $J=7.3 \mathrm{~Hz}$ ), t $6.181 \mathrm{ppm}$; d 6.130 ppm; d 5.609 ppm; q 4.398 ppm, q 4.188 ppm; s 1.9 ppm, as shown in Fig. S1. $\dagger$

2.2.2. Synthesis of e-ATRP agents. $0.1 \mathrm{~g}$ of MWCNT/ graphene/SWCNH derivatized with a $p$-aminoaniline were dispersed in $10 \mathrm{~mL}$ of dry $\mathrm{CHCl}_{3}$ and cooled down to $0{ }^{\circ} \mathrm{C}$ in a $100 \mathrm{~mL}$ Schlenk flask charged with a stirring bar in an ice/salt bath. ${ }^{16}$ Then a 2-bromo-2-methylpropionyl bromide ( $0.3 \mathrm{~g}, 0.013$ mmol $)$, triethylamine $(0.2 \mathrm{~mL}, 1.43 \mathrm{mmol})$ and $N, N$-dimethylaminopyridine (DMAP, $0.02 \mathrm{~g}, 0.164 \mathrm{mmol}$, dissolved prior the addition in $1.0 \mathrm{~mL}$ dry $\mathrm{CHCl}_{3}$ ) were added via a cannula transfer to the flask and the reaction temperature was maintained at $0{ }^{\circ} \mathrm{C}$ for $1 \mathrm{~h}$, and then for $48 \mathrm{~h}$ at room temperature. Afterwards the solid was separated from the reaction mixture by suction filtration under air and washed five times with $20 \mathrm{~mL}$ of dry $\mathrm{CHCl}_{3}$. The black solids were collected and dried in the air pressure oven at $40{ }^{\circ} \mathrm{C}$. An approximately $0.098 \mathrm{~g}$ of initiators were collected in each case (MWCNT, graphene and SWCNH) and analyzed by FTIR, Raman spectroscopy and EDX (Fig. S2 and $\mathrm{S} 6 \boldsymbol{\dagger}^{\dagger}$.

2.2.3. Preparation of RAFT agents. $0.2 \mathrm{~g}$ MWCNT-benzoyl acid chloride, graphene-benzoyl acid chloride, SWCNH-acid chloride synthesized according to the procedure described before ${ }^{\mathbf{1 6}}$ were placed in a $50 \mathrm{~mL}$ Schlenk flask charged with the magnetic stirrer; under dry nitrogen (by standard five-time vacuum/nitrogen purging). A $10 \mathrm{~mL}$ of dry THF was introduced via three times evacuated glass syringe. A pre-synthesized dithiopropanoic magnesium chloride salt $(6.4 \mathrm{~mL}, 1.64 \mathrm{~mol}$ in $\mathrm{THF}$ ) was introduced to the reaction flask at $60^{\circ} \mathrm{C}$ via a cannula 


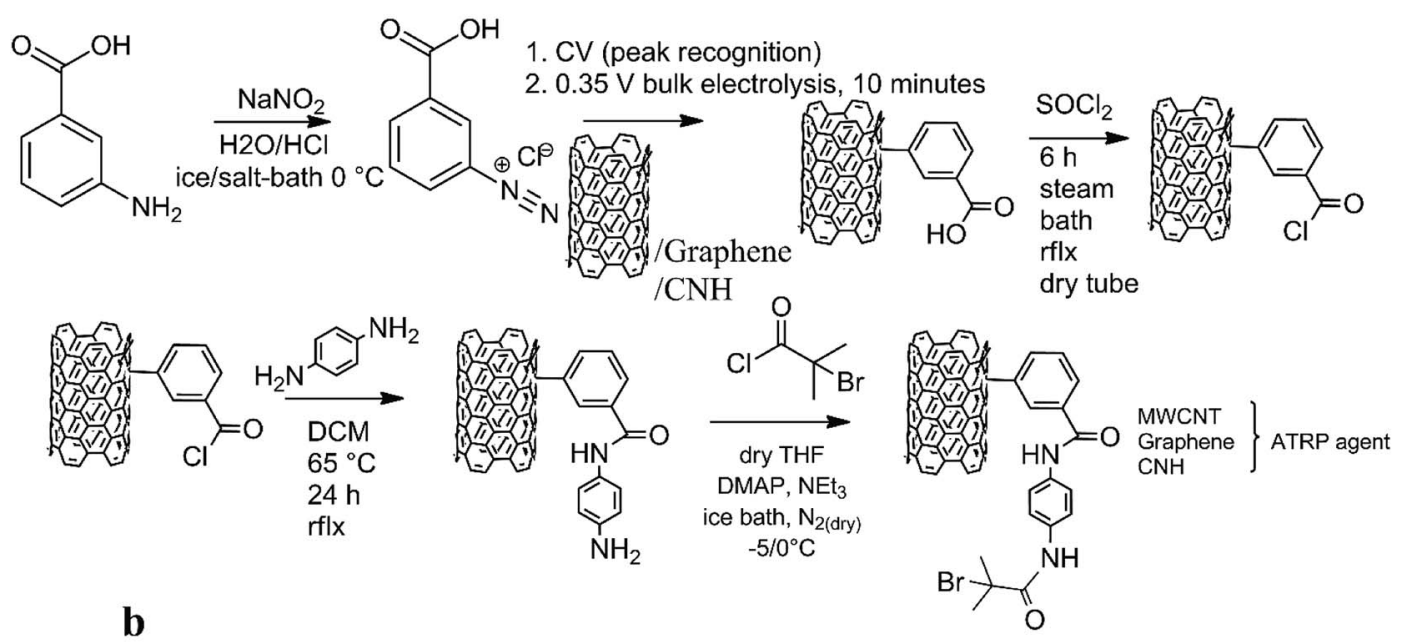

.
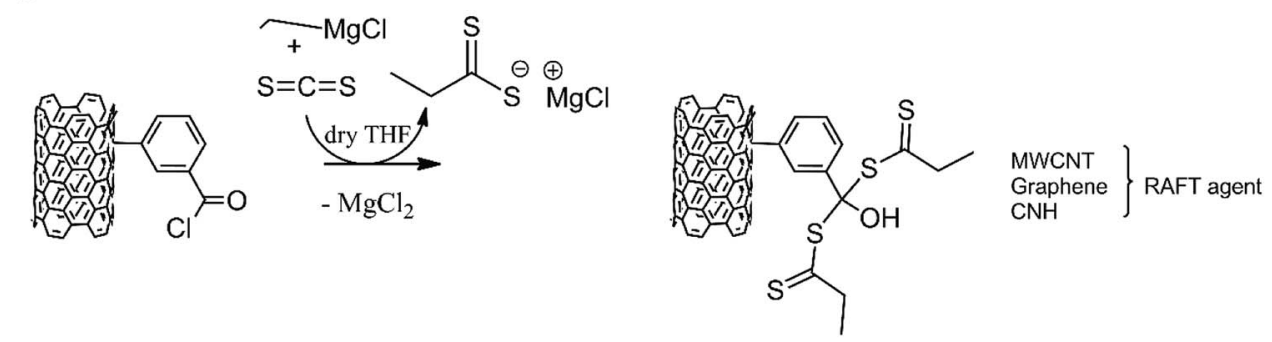

C

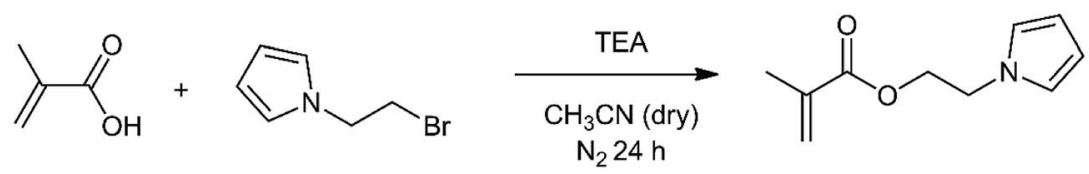

Fig. 1 The preparation of carbon-grafted initiators ( $a$ and b) and a 2-(1H-pyrrol-1-yl)ethyl methacrylate monomer (c).

transfer under the counter stream of dry nitrogen. The reaction was carried out for 24 hours, the solid product was collected by suction filtration under air and washed five times with dry THF. After drying at $40^{\circ} \mathrm{C}$ under vacuum, $\sim 0.12 \mathrm{~g}$ of respective RAFTagent was obtained in each case. Fig. S2 $\uparrow$ shows the Raman analysis of selected synthesized RAFT agents and the signals observed were consistent with literature. ${ }^{22}$ Examples of FTIR (a) and Raman spectra (b) for the MWCN-based RAFT initiator are presented in Fig. 2. The FTIR signals at $1300 \mathrm{~cm}^{-1}$ are assigned to $\mathrm{SC}=\mathrm{S}$ asymmetric stretch, $1070 \mathrm{~cm}^{-1}$ for the $\mathrm{S}-\mathrm{C}=\mathrm{S}$, or typical free-OH symmetric stretch. ${ }^{22}$ Small discrepancies such as the transmittance shift of $2 \mathrm{~cm}^{-1}\left(3697 \mathrm{~cm}^{-1}\right.$ reported, in case of this study $3699 \mathrm{~cm}^{-1}$ (ref. 22)) for the free $-\mathrm{OH}$ stretch could be due to the presence of an additional aryl unit used to prepare the RAFT initiator. Fig. $2 \mathrm{~b}$ shows the Raman spectrum of the functionalized MWCNTs that consists three main peaks. The signal at $1304.23 \mathrm{~cm}^{-1}$ is assigned to defects and disordered graphite structures, while the peaks at $1598.36 \mathrm{~cm}^{-1}$ and $2601.46 \mathrm{~cm}^{-1}$ are attributed to the graphite band, which is common to all $\mathrm{sp}^{2}$ systems and second-order Raman scattering process, respectively. The ratio of intensity for the defect band and graphite band $\left(I_{\mathrm{D}} / I_{\mathrm{G}}\right)$ indicates on the degree of functionalization of the MWCNTs. ${ }^{23}$ For a pure MWCNT, the $I_{\mathrm{D}} / I_{\mathrm{G}}$ observed in this work was 1.91 and, as can be estimated from Fig. $2 \mathrm{~b}, I_{\mathrm{D}} / I_{\mathrm{G}}$ of functionalized carbon nanotubes is 1.87 , demonstrating that $2.08 \%$ of the carbon has been functionalized in the MWCNT-based RAFT agent. The similar analysis of the $I_{\mathrm{D}} / I_{\mathrm{G}}$ intensity ratio for the graphene- and SWCNH-based RAFT reagents were carried from spectra demonstrated in Fig. S2 (ESI $\dagger$ ), and revealed degree of carbon functionalization similar to that of MWCNTs.

2.2.4. Synthesis of dithiopropanoic magnesium chloride. $5.25 \mathrm{~mL}$ of $2 \mathrm{~mol}$ ethyl magnesium chloride in THF was transferred to $25 \mathrm{~mL}$ Schlenk flask under dry nitrogen in the glove bag. Afterwards, $1.15 \mathrm{~mL}$ of dry carbon disulfide $(0.15 \mathrm{mmol})$ was introduced via a three-time evacuated glass syringe to the ethyl magnesium chloride solution dropwise over 5 minutes. The change of color from clear brown to pale red was observed and was also consistent with literature. ${ }^{23}$ The formation of product was confirmed by ${ }^{1} \mathrm{H}$ NMR (Fig. S4, ESI $\dagger$ ). After 1 hour of stirring at room temperature, the obtained product was used immediately without further purification.

2.2.5. e-ATRP polymerization. $0.217 \mathrm{~g}$ of a tetra- $n$-butylammonium tetrafluoroborate $\left(\mathrm{TEABF}_{4}\right)$ in $10 \mathrm{~mL}$ of dry $\mathrm{CH}_{3} \mathrm{CN}$ was purged with nitrogen in a three-neck electrochemical cell for 30 minutes. In next step, $0.217 \mathrm{~g}(0.17 \mathrm{~mL})$ of $0.0724 \mathrm{~mol}$ (in 


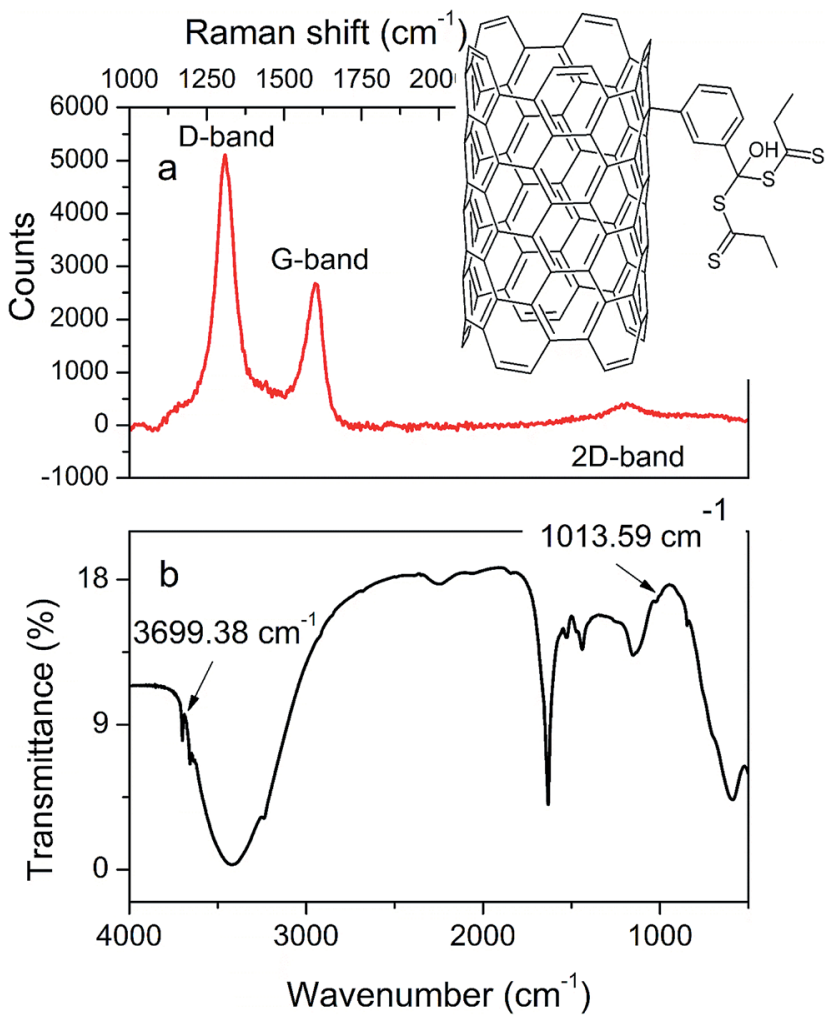

Fig. 2 The Raman spectra (a) and FTIR (b) for the MWCNT-based RAFT agent.

dry $\mathrm{CH}_{3} \mathrm{CN}$ ) freshly prepared $\mathrm{Cu}(\mathrm{I}) \mathrm{Br} / \mathrm{PMDETA}$ complex was added to the solution under stirring, and several cyclic voltammograms were recorded. The pre-mixed $0.05 \mathrm{~mL}$ of a $2-\left(1 \mathrm{H}^{-}\right.$ pyrrol-1-yl)ethyl methacrylate was combined with a $0.01 \mathrm{~g}$ of an ATRP initiator. Under a constant recording of CV scans, the electrochemically-assisted reaction was carried out for 240 minutes and the conversion of monomer was confirmed by ${ }^{1} \mathrm{H}$ NMR $\left(\mathrm{CDCl}_{3}\right)$. The color changed from green to blue and the precipitation of carbon allotropes grafted with the polymer was observed due to the increase in the mass of the product (Fig. S5, ESI $\dagger$ ). The product was collected by suction filtration, washed with dry $\mathrm{CH}_{3} \mathrm{CN}$ and subjected to crosslinking with the pyrrole monomer in the presence of ammonium persulfate according to the procedure reported previously. ${ }^{14}$ The e-ATRP electrochemical setup utilized in this synthesis is shown in Fig. S5. $\dagger$ The voltammetry parameters for the polymerization were adapted from literature ${ }^{25,26}$ An EDX elemental analysis was first screening tool that confirmed the presence of e-ATRP product (and exemplary EDS spectra is shown in Fig. S6 in ESI †).

2.2.6. RAFT polymerization. $0.011 \mathrm{~g}$ of the chain transfer RAFT agent was placed in a $50 \mathrm{~mL}$ Schlenk flask charged with a magnetic stirrer alongside with $0.00275 \mathrm{~g}(0.017 \mathrm{mmol})$ AIBN and $0.065 \mathrm{~g}$ of a $2-(1 \mathrm{H}$-pyrrol-1-yl)ethyl methacrylate. $20 \mathrm{~mL}$ of dry DMF were introduced and heated at $65^{\circ} \mathrm{C}$ under continuous magnetic stirring overnight. In order to observe the conversion of a monomer in time, ${ }^{1} \mathrm{H}$ NMR samples in $\mathrm{CDCl}_{3}$ were taken after 30, 60, 120, 150, 180 and 210 minutes. The brown product was separated from dark green reaction mixture by suction filtration and washed with $\mathrm{CHCl}_{3}$ (wet) till the filtrate was colorless. The crosslinking of the pyrrole in the presence of ammonium persulfate was performed according to the procedure described previously. ${ }^{14}$ The obtained RAFT products were analyzed by FTIR, Raman spectroscopy and EDS elemental analysis (and examples of EDS spectra are shown in ESI in Fig. S7 and S8†).

\section{Results and discussion}

\subsection{Electrochemical study on e-ATRP polymerization process and RAFT synthesis}

Fig. 3 a shows the synthesis steps in e-ATRP process, which begins from the electrochemical activation of a pentamethyldiethyltriamine (PMDETA) copper(I) bromide complex used as a co-initiator, and synthetized according to referred work. ${ }^{27}$ The electrochemically aided reduction of copper is observed at $E_{1 / 2}=-0.85 \mathrm{~V}$, and is consistent with literature $\left(E_{1 / 2}\right.$ $=-0.72 \mathrm{~V},{ }^{28}$ Fig. S13 in ESI $\dagger$ demonstrates an exemplary $\mathrm{CV}$ scan of $\mathrm{Cu}(\mathrm{I})$ reduction). The $\mathrm{Cu}$ complex is core species resulting in activity of the co-initiator in the e-ATRP. The activation and deactivation constants of this process can be calculated based on analysis of Butler-Volmer equation (eqn (1)) derived from the Tafel plot, $\eta=f(\log i)$ (Fig. S9 in ESI $\dagger$ ):

$$
k_{\text {act }}=k_{0} \exp \left[\left(-\frac{\alpha n F}{R T}\left(E-E_{0}\right)\right)\right] .
$$

The $k_{\text {act }}$ is the reaction rate for forward reaction (also called activation rate constant), $k_{0}$ is the equilibrium reaction constant, $\alpha$ denotes the electron transfer coefficient obtained from the Tafel plot (Fig. S9†), $n$ is the number of electrons transferred during the reaction, $F$ is the Faraday constant (96 $\left.485.33 \mathrm{C} \mathrm{mol}^{-1}\right), R$ is the universal gas constant $(8.314 \mathrm{~J}$ $\left.\mathrm{mol}^{-1} \mathrm{~K}^{-1}\right), T$ is temperature $(295.65 \mathrm{~K}$ at the time of measurement), $E$ and $E_{0}$ are applied potential and the standard potential for the reduction reaction, respectively. The slope of Tafel function for the cathodic activation process is equal to $\frac{(1-\alpha) F}{2.3 R T}$. Briefly, when linear branch of the cathodic Tafel plot (Fig. S9 $\dagger$ ) is extrapolated to $\log (i)$, the steady-state equilibrium for the reduction/oxidation reaction responsible for the catalyst activation is reached at the respective exchange current density, $i_{0}$ (e.g., for MWCNT-based initiator $i_{0}=5.38 \times 10^{-6} \mathrm{~A}$ and is recalculated from the common logarithm with the base of 10$)$. This quantity is further used to calculate the equilibrium $\left(k_{0}\right)$ and $\left(k_{\text {act }}\right)$ in eqn (1) $\left(k^{0}=\frac{i_{0}}{F A C}\right.$ at the complex concentration of $C$ $\left.=1.1 \times 10^{-3} \mathrm{~mol} \mathrm{~cm}^{-3}\right)$. The overpotential observed for the eATRP assisted activation depends on the type of carbon allotrope used in the synthesis, as shown in Tafel plots (Fig. S9†). As a result, the calculated activation constant $\left(k_{\text {act }}\right)$ varies for all eATRP products as follow: $0.023 \mathrm{~L} \mathrm{~mol}^{-1} \mathrm{~s}^{-1}$ for the MWCNTbased initiator, $0.261 \mathrm{~L} \mathrm{~mol}^{-1} \mathrm{~s}^{-1}$ for the graphene-based and $0.840 \mathrm{~L} \mathrm{~mol}^{-1} \mathrm{~s}^{-1}$ for the SWCNH-based initiator, and slightly differ from the activation constant calculated using the same method applied to similar systems. ${ }^{27,28}$ In this work, the 

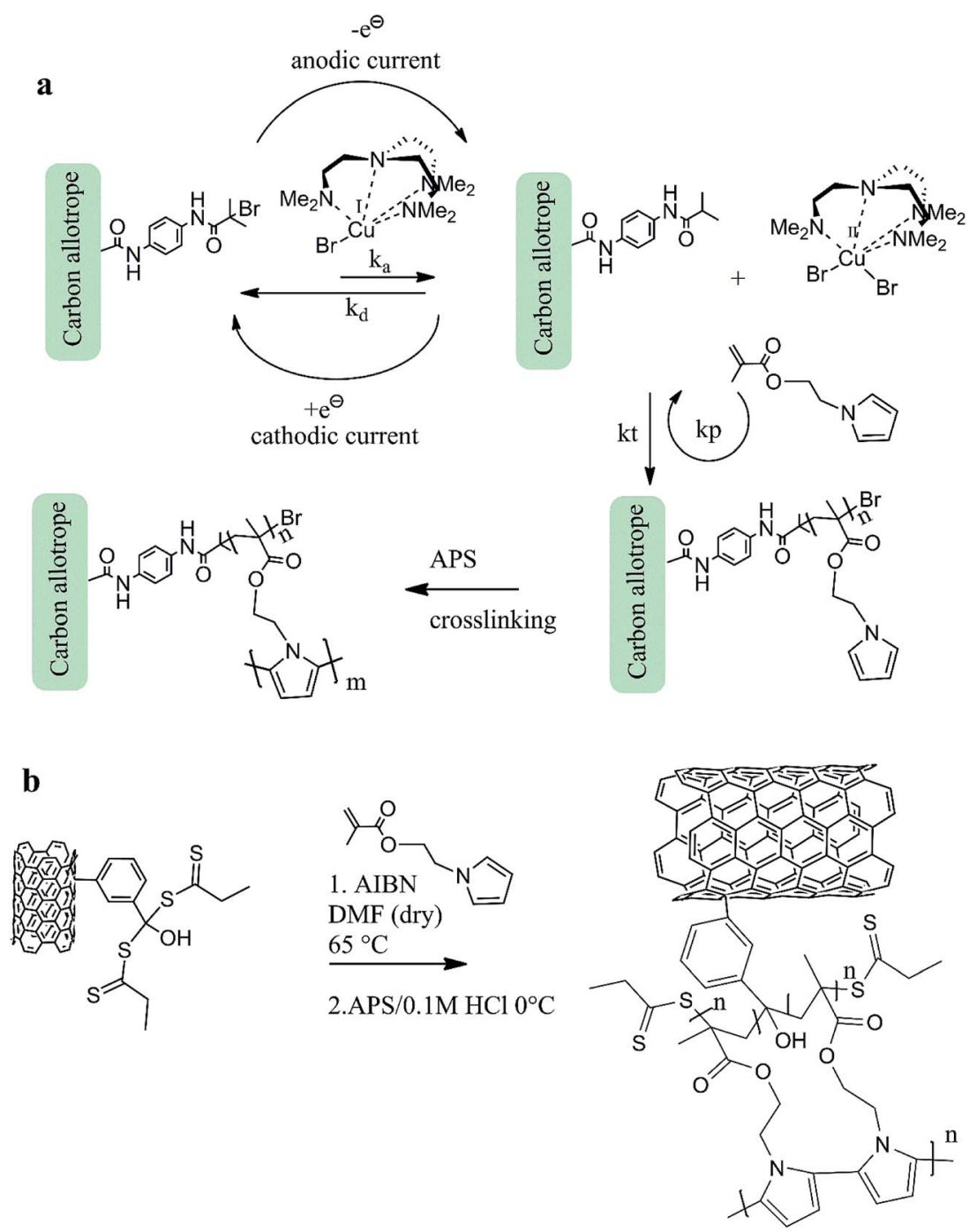

Fig. 3 The synthesis steps in e-ATRP (a) and the RAFT (b) polymerization of the carbon-grafted-polymer capacitor materials.

SWCNHs-derived initiator showed the highest $k_{\text {act }}$ and the lowest overpotential needed to initiate the redox process, as compared to other allotropes. This difference is apparently related to the carbon specific surface area, which is the highest for the SWCNHs. Thus, the accessibility of electrochemical interface for the redox process is largest in SWCNHs. The particle size and shape for carbons investigated in this work are very different, therefore the distributions of the electro-active centers along the carbon three-dimensional $\pi$-extended systems varies accordingly. The porous structure of SWCNs and their smallest size as compared to the graphene and MWCNT initiators, caused the fastest $\mathrm{Cu}$ redox activity on SWCNH. On the other, an open planar structure of the graphene sheets (not structurally hindered) also facilitates fast and easy transport and activity of $\mathrm{Cu}$ complex, and thus the $k_{\text {act }}$ calculated for the graphene is also high and comparable to carbon-based initiators reported in literature. ${ }^{29,30}$ Nevertheless, the efficiency of $\mathrm{Cu}$ activation in the e-ATRP process is strongly influenced by the carbon morphology.
Fig. $2 \mathrm{~b}$ shows the synthesis steps in the RAFT polymerization, partially adapted from literature. ${ }^{24}$ The molar ratios of AIBN/initiator/monomer were kept at $1: 4: 24$. The RAFT synthesis has some advantages over e-ATRP, such it does not require electrochemical instrumentation. Since the e-ATRP uses reference electrode, it is critical to prevent the synthesis workup from any contamination from reference electrode leakage. Also, the RAFT polymerization does not require a toxic copper complex, and thus the overall workup is easier than in e-ATRP (where copper removal is an additional step in purification of the product). On the other hand, the e-ATRP allows to perform the subsequent electro-polymerization of the pyrrole units using the same electrochemical setup, in total, the synthesis can be faster. More importantly, in e-ATRP, an in situ analysis of kinetics of the polymer growth can be carried out using wellknown electrochemical models (such as the Butler-Volmer and Tafel analysis). The RAFT is a pure chemical synthesis, whereby the e-ATRP combines the electrochemical approach with typical chemical methods. Nevertheless, the advantages of 
both synthesis routes are discussed in context of composites' morphology, the electrochemical stability and energy storage capacity in following sections.

\subsection{Spectroscopic analysis of the carbon-grafted polymers}

Fig. 4 demonstrates FTIR (a-c) and Raman spectra (d-f) of the carbon-grafted-polypyrrole capacitive materials. The most significant differences in FTIR spectra appear for the end-group of the carbon allotrope based RAFT initiators as a results of different polymerization approaches. A free $-\mathrm{OH}$ stretch at $3700 \mathrm{~cm}^{-1}$ corresponding to the aryl unit attached to the multi-walled carbon nanotube indicates on the presence of RAFT agent. This moiety is composed of a dithiopropanoic aryl magnesium salt, ${ }^{24}$ as shown in Fig. 4a for the MWCNT-grafted-PPy synthetized by RAFT. The same stretching band at $3697 \mathrm{~cm}^{-1}$ is reported by other group for the MWCNT-based initiator. ${ }^{24}$ Similar signals with minor shifts are observed for the graphene and SWCNH initiators (Fig. S2 $\dagger$ ). The following FTIR bands identify the final product: a weak-medium stretch band at $571 \mathrm{~cm}^{-1}$ arises from the presence of aromatic $=\mathrm{C}-\mathrm{H}$ deformation vibration in the initiator's aryl unit; at 753 $\mathrm{cm}^{-1}$ the $\mathrm{C}-\mathrm{S}$ strong vibration indicates on the presence of thioester, a medium C-O-C vibration at $808 \mathrm{~cm}^{-1}$ corresponds to the methacrylate backbone, esters relate to bands visible at 1190 and $1250 \mathrm{~cm}^{-1}$, a large carbonyl stretch at $1740 \mathrm{~cm}^{-1}$ indicates the presence of the meta acrylic backbone in RAFT product. Also, a large band at $1650 \mathrm{~cm}^{-1}$ is assigned to $-\mathrm{C}=\mathrm{C}-$ aromatic vibrations from MWCNTs and an aryl functionality of meta-substituted benzene ring, strong stretching vibrations at $1400 \mathrm{~cm}^{-1}$ and 1450 $\mathrm{cm}^{-1}$ alongside with the broad weak band at 3000 and $3420 \mathrm{~cm}^{-1}$ confirm the presence of poly(pyrrole) unit. Also, the $=\mathrm{C}-\mathrm{H}$ and $\mathrm{C}=\mathrm{N} /-\mathrm{C}-\mathrm{N}$ - in plane vibrations are typically observed in the 1substituted pyrroles. ${ }^{29}$ The $\mathrm{C}-\mathrm{H}$ aliphatic bending vibrations are present at $2950 \mathrm{~cm}^{-1}$. In total, the most important signals revealing the formation of composites are: the free $-\mathrm{OH}$ stretch at $3700 \mathrm{~cm}^{-1}$ for the initiator derivatized with the 3-substituted aryl mote; the resolved weak $1-\mathrm{H}$ isolated deformation vibration of the 3-substituted aryl attached to the MWCNT core at $708 \mathrm{~cm}^{-1}$, and corresponding the 3-H atoms with the out-of-plane deformation vibrations at $835 \mathrm{~cm}^{-1}$; the strong dithionic $\mathrm{C}=\mathrm{S}$ and $\mathrm{C}-\mathrm{S}$ alongside with the $\mathrm{C}-\mathrm{C}$ vibrations from this functionality observed at 1120, 982 and $1150 \mathrm{~cm}^{-1}$; two bands at 891 and $948 \mathrm{~cm}^{-1}$ represent dithionic units in the endcapped polymer backbone around the carbon core (which was also confirmed by the sulfur signal observed in EDS spectrum Fig. S6†). The Raman analysis of the MWCNT-grafted-PPy (Fig. 4d) shows characteristic for the carbon nanotube, the $D$ and $G$ bands (at 1311.85 and $1610.19 \mathrm{~cm}^{-1}$, respectively). The analysis of $\mathrm{D} / \mathrm{G}$ intensity ratio related to the pristine MWCNT is an indication on the extent of the surface derivatization and is related to the change of $\mathrm{sp}^{2}$ hybridized carbon atoms to $\mathrm{sp}^{3}$ after the attachment of the linker. This analysis is done based on the comparison of the ratio of $\mathrm{D} / \mathrm{G}$ in pristine MWCNT and the same carbon in composite (according to relation: $\left.100-\left(\left(\mathrm{D} / \mathrm{G}_{\text {MWCNT-based initiator }}\right) /\left(\mathrm{D} / \mathrm{G}_{\text {pristine MWCNT }}\right) \times 100 \%\right)\right)$. For the MWCNT-grafted-PPy the $\mathrm{D} / \mathrm{G}$ ratio was 1.68 , and for pristine MWCNT it was 1.91 , which corresponds to $12.04 \%$ of the carbon surface functionalization with the linker and polymer. The D/G ratio from MWCNT- $g$-PPy prepared by RAFT polymerization was
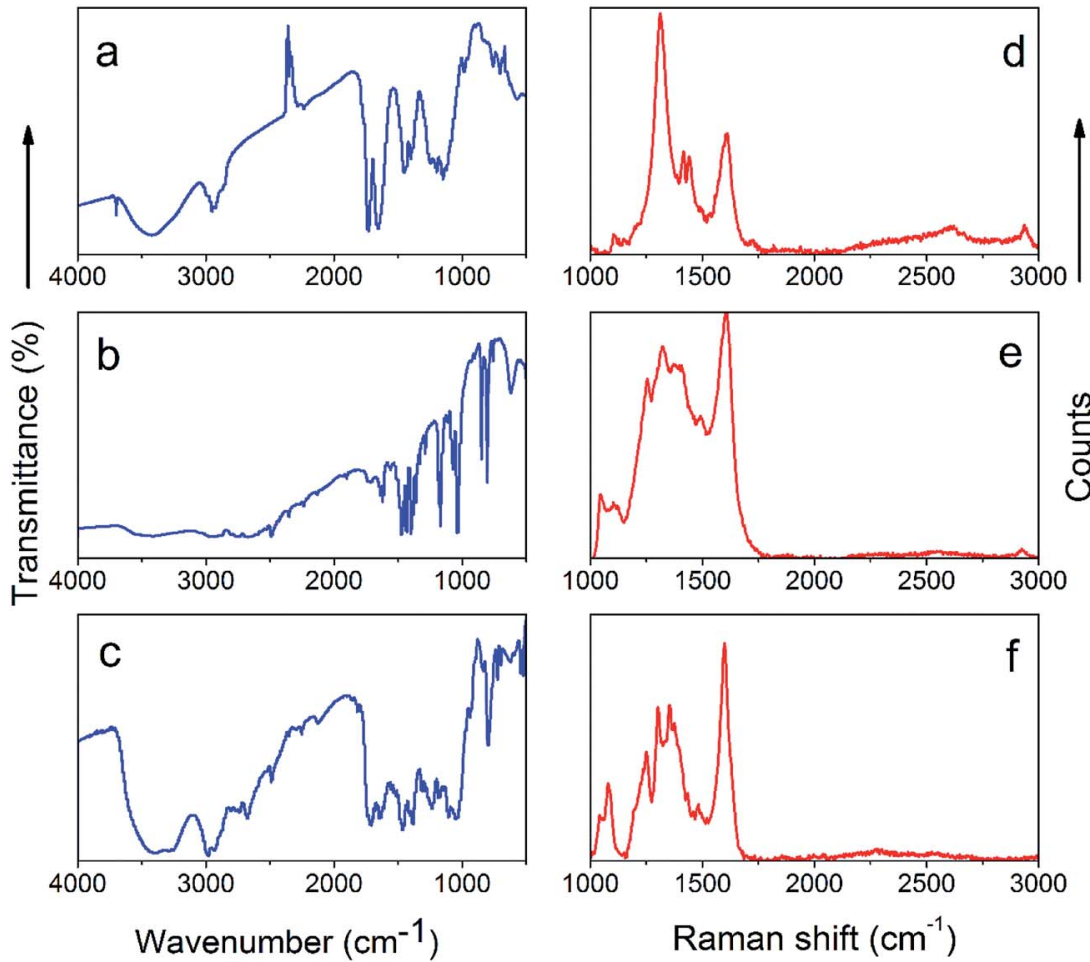

Fig. 4 FTIR $(a-c)$ and Raman spectra ( $d-f)$ of e-ATRP and RAFT products: MWCNTs-grafted-PPy by RAFT (a and d), SWCNH-grafted-PPy by eATRP ( $b$ and e), graphene-grafted-PPy by e-ATRP ( $c$ and $f$ ). 
2.015. The increase in the $\mathrm{D} / \mathrm{G}$ intensity ratio, is due to combined effects of the increase in intensity of $\mathrm{sp}^{3}$ hybridized atoms as compared to the $\mathrm{sp}^{2}$, alongside with the influence of the length of methacrylate backbone (due to the rich abundance of $\mathrm{sp}^{3}$ carbon atoms in their structure). The $\mathrm{D} / \mathrm{G}$ ratio for pristine graphene was 0.69 (which correlates well with large abundance of $\mathrm{sp}^{2}$ hybridized carbon atoms) and for pristine single-walled carbon nanohorns 1.88 (data not shown). For SWCNH- and graphene-based initiators, the $\mathrm{D} / \mathrm{G}$ ratios were 1.22 and 0.48 , respectively, which corresponds to 30 and $35 \%$ abundance of functional groups onto the carbon surface. For the MWCNT-grafted-PPy prepared by the RAFT (Fig. 4d) there are additional peaks at 1104.76, 1417.70, 1441.61, 2605.72 and $2935.49 \mathrm{~cm}^{-1}$ assigned to the $-\mathrm{CH}_{2}-$ aliphatic chain and the poly(pyrrole) units with a $2 \mathrm{D}$ band at $2935.49 \mathrm{~cm}^{-1}$ and also associated with the broadening of $\mathrm{sp}^{2}$ signals due to covering of carbon with the polymer. This results in the splitting phonon band from one $\mathrm{sp}^{2}$-rich layer of carbon core to other $\mathrm{sp}^{2}$-rich layer of the poly(pyrrole).$^{31,32}$

Fig. $4 \mathrm{~b}$ and c demonstrate the FTIR spectra of the SWCNHgrafted-PPy and graphene-grafted-PPy synthesized by e-ATRP, respectively. For the first composite, the characteristic signal indicating on the product formation is a band at $522 \mathrm{~cm}^{-1}$ assigned to an out of plane $\mathrm{N}-\mathrm{H}$ deformation vibration in amide structure, which correlates with the second band at $542 \mathrm{~cm}^{-1}$. The moderate intensity $\mathrm{C}-\mathrm{O}$ stretching amide vibration is observed at $1040 \mathrm{~cm}^{-1}$ and at $1170 \mathrm{~cm}^{-1}$ that are similar to bands found in amides connected directly to aryl groups, for example in carbons coupled with a $p$-amino aniline. ${ }^{\mathbf{1 6}}$ The medium and strong in-plane $\mathrm{N}-\mathrm{H}$ bending vibrations assigned to the amide bond are seen at 1320 (medium) and $1570 \mathrm{~cm}^{-1}$ (strong). The band at $585 \mathrm{~cm}^{-1}$ is attributed to $\mathrm{CH}_{2}$ vibration of the ethyl unit in ester bond between the pyrrole and methacrylate. The band at $617 \mathrm{~cm}^{-1}$ correlated with the band at 1240 $\mathrm{cm}^{-1}$ and both are assigned to the aliphatic $\mathrm{C}-\mathrm{Br}$ ending of the polymer methacrylic backbone. Similar to the MWCNT-graftedPPy prepared by RAFT (Fig. 4a), the peaks at 724 and $832 \mathrm{~cm}^{-1}$ indicate on the presence of the meta-substituted aryl mote. The bands at 669 and $698 \mathrm{~cm}^{-1}$ are attributed to the $\mathrm{C}-\mathrm{H}$ wagging vibrations of an ethyl aliphatic unit, connecting PPy to the methacrylate. The bands at 745, 762 and $781 \mathrm{~cm}^{-1}$ arise from the $\mathrm{N}-\mathrm{H}$ wagging vibrations. The bands at $621,941,969$ and $1000 \mathrm{~cm}^{-1}$ recognize the presence of the $\mathrm{N}-\mathrm{C}$ and $\mathrm{C}-\mathrm{H}$ deformation vibrations in the $1-\mathrm{H}$ substituted pyrroles. The bands at 1030 and $1320 \mathrm{~cm}^{-1}$ are assigned to the $\mathrm{C}-\mathrm{O}$ vibration in ester unit that correlates well with the carbonyl stretch at $1720 \mathrm{~cm}^{-1}$. The presence of poly(pyrrole) unit is additionally confirmed by bands at 1060, 1080 and $1470 \mathrm{~cm}^{-1}$. The aryl units manifest themselves by the band at $1640 \mathrm{~cm}^{-1}$ alongside with the presence of large aromatic graphene sheets. The aliphatic chain at 2050 and $2800 \mathrm{~cm}^{-1}$ almost overlap with the $\mathrm{N}-\mathrm{H}$ signals from the amides at 2850 and $2930 \mathrm{~cm}^{-1}$. The final amide and ester structures are confirmed by the bands at 3260 and $3420 \mathrm{~cm}^{-1}$.

Raman spectroscopy of the graphene-grafted-PPy (Fig. 4f) revealed stronger $\mathrm{D}$ band at 1250 , and $2 \mathrm{D}$ at $2271 \mathrm{~cm}^{-1}$ corresponding to the phonon splitting in case of the MWCNTgrafted-PPy prepared by RAFT. The difference between these
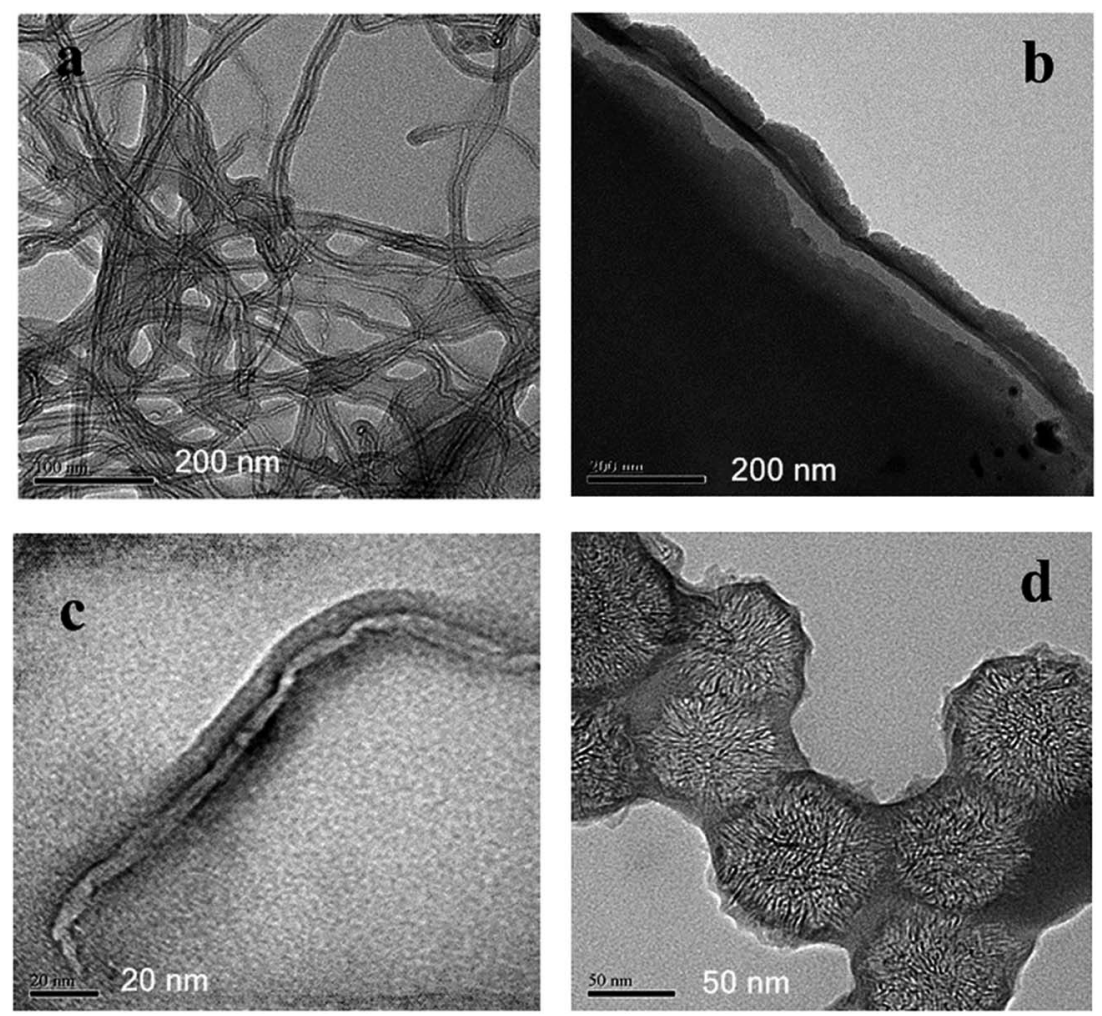

Fig. 5 TEM images of e-ATRP and RAFT products. MWCNT- $g$-PPy by e-ATRP (a), graphene- $g$-PPy by RAFT (b), MWCNT- $g$-PPy by RAFT (c) and SWCNH-g-PPy by e-ATRP (d). The bare carbon allotropes are shown in Fig. S10-S12 in ESI. $\dagger$ 
two composites is only the intensity of the signal that is stronger in graphene due to the presence of approximately twelve sheets that overlay and form a sandwich-like structure (based on TEM imaging, Fig. 5), which contributes to higher intensity of D signal as well as stronger $\mathrm{G}$ band at $1352 \mathrm{~cm}^{-1}$. Signals at 1042 , 1081, 131, 1376, 1434, 1460, 1480, 1579 and $1600 \mathrm{~cm}^{-1}$ correspond to the PPy unit with covalent linkers and the methacrylic backbone. For example, peaks at 1042 and $1081 \mathrm{~cm}^{-1}$ are associated with the phenyl ring and the peak at $1301 \mathrm{~cm}^{-1}$ arises from the $\mathrm{C}=\mathrm{C}$ alkyl stretch. The ester units are identified by the presence of COO- stretch at $1376 \mathrm{~cm}^{-1}$ and at $1434 \mathrm{~cm}^{-1}$ assigned to the stretching of methacrylic backbone. The latest is also confirmed by the presence of $-\mathrm{CH}_{3}$ stretch at $1460 \mathrm{~cm}^{-1}$. The poly(pyrrole) shows additional stretching signals at 1480 and $1519 \mathrm{~cm}^{-1}$. The Raman spectra of the SWCNH-grafted-PPy (Fig. 4e) and the MWCNT-grafted-PPy (Fig. 4d) demonstrate similar signals with lower intensities.

\subsection{Morphology}

TEM images of selected final products are shown in Fig. 5. The structure of carbon allotropes chosen in this study are very different, and thus the morphology of composites is specific to the type of carbon. The general observation is that materials synthetized by both RAFT and e-ATRP methods show very thin polymer layer along the carbon for all cases. This can be seen by comparing the MWCNT-grafted-PPy made by e-ATRP (Fig. 5a) and the MWCNT-grafted-PPy by RAFT (Fig. 5c). Both living polymerization techniques allow control over the poly(pyrrole) particle size or its film thickness, which are in the range of nanometers for all products. The PPy structure is critical aspect of its electrochemical activity, and should be optimized in order to facilitate a charge transfer during doping-dedoping of polymer chain (pseudo-capacitance activity). Also, the SWCNHgrafted-PPy made by e-ATRP (Fig. 5d) shows a non-continuous polymer, which should further benefit in a high double-layer capacitance from the carbon exposed to the electrolyte. The completely different structure is observed for the graphenebased composite synthesized by RAFT (Fig. 5b), where several graphene layers are sandwiched between very thin PPy films. Both, the carbon and PPy exposed to the electrochemical interface benefit in synergistic improvement of capacitance due to efficient double-layer and pseudo-capacitance. The morphology of final products is the most critical parameter that affects the electrochemical activity and stability of these composites and will be further discussed in conjunction with electrochemical tests.

\subsection{Electrochemical analysis: charge-discharge, mass transfer, long-term cycle stability}

The redox activity of poly(pyrrole) in faradaic region and double layer capacitance of the carbon can be observed by the cyclic voltammetry (Fig. 6 and 7). In the potential range of anodic activity of the polymer (shown as a broad oxidation wave with a weak oxidation signals, Fig. 6b), a positively charged radical

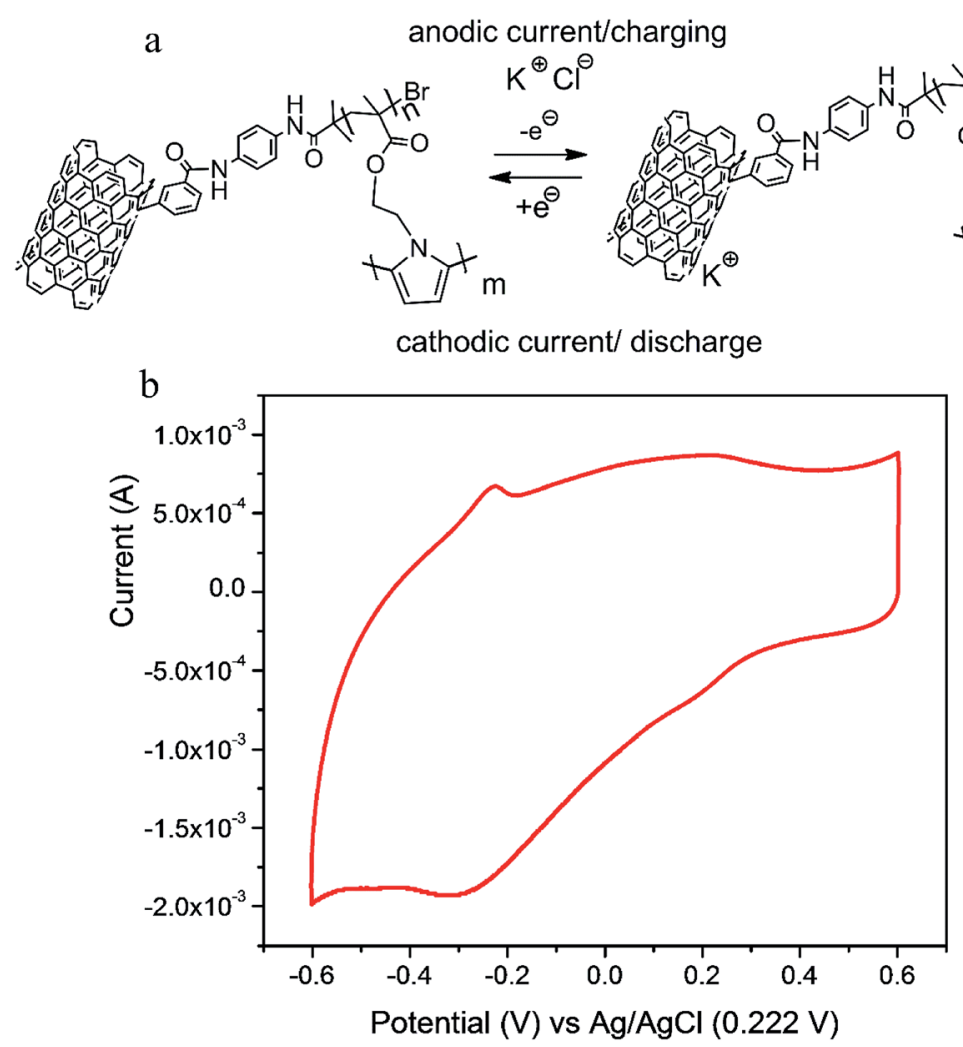

Fig. 6 The redox activity of poly(pyrrole) generating pseudo-capacitance (a), an example of the cyclic voltammogram of the MWCNT-graftedPPy synthetized by e-ATRP (b). 
cation is created due to the stripping of an electron from lone pair on nitrogen, as shown in Fig. 6a. In order to compensate the deficiency of negative charge, a chloride from the electrolyte contributes in doping of poly(pyrrole). The process is highly reversible, and soon after the reverse scan is applied, the reduction of polymer chain occurs via formation of neutral species (Fig. 6a). The nitrogen lone pair becomes neutral and the doping anion is expelled to solution. The redox activity (and associated doping) is efficient only if the process occurs within the polymer bulk. Therefore, the polymer films should be very thin, or the particle size in case of powders - very small.

The repetitive charge-discharge of poly(pyrrole) introduces considerable volumetric changes in the polymer. This feature of conjugated polymers makes them good actuators (e.g., for the application in artificial muscles). However, this is also a reason for their electrochemical instability. For example, comparing the poly(pyrrole) with poly(aniline), the conductivity in the latter (generated via anion doping and the cation formation in oxidized state) is achieved upon the linearization of phenyl structure that requires additional energy. ${ }^{32,33}$ The poly(pyrrole) is a chain molecule composed of five-membered heterocycles, and it has better stability as compared to the poly(aniline) due to accommodation of nitrogen in the ring structure. ${ }^{34}$ Such structure of poly(pyrrole) hampers the formation of destructive polaron and multi-polaron. ${ }^{34}$ The electronic stabilization of the polymer via its grafting with carbons, and generated by this electronic effects, such as the reverse donor-acceptor centers, yield higher electrochemical stability. ${ }^{14}$

In presented study we report the values of gravimetric capacitance and other electrochemical parameters only for the

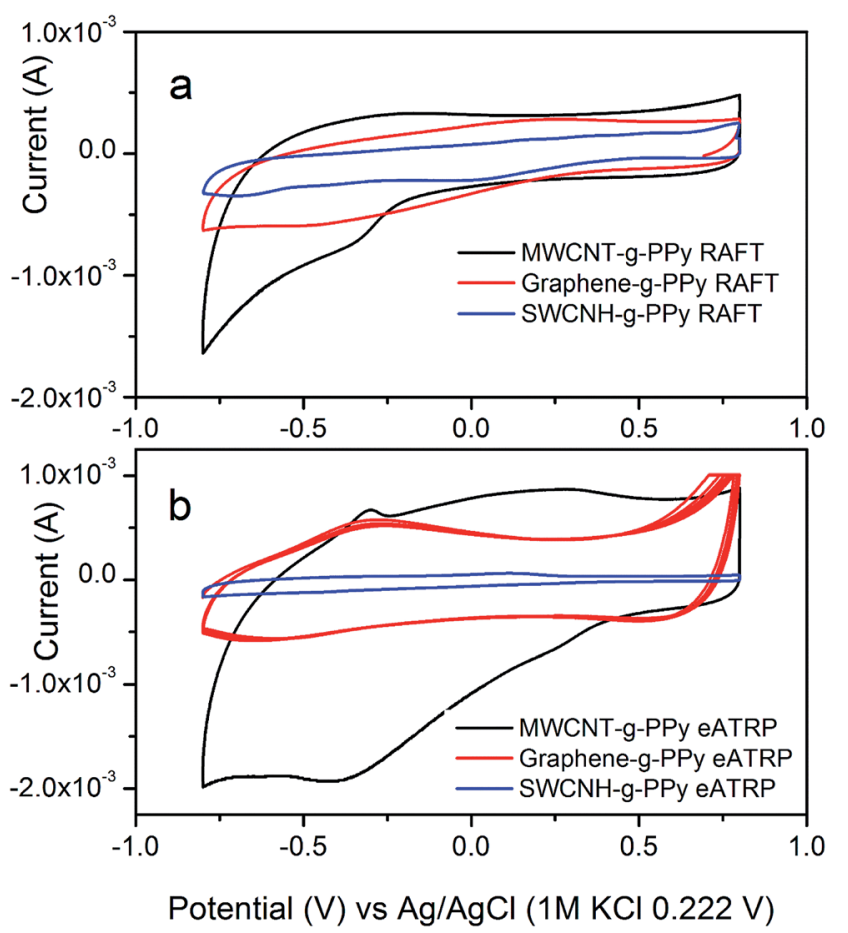

Fig. 7 Cyclic voltammograms of carbon allotropes grafted with poly(pyrrole) prepared by RAFT (a) and e-ATRP (b). final products. The intermediate products (oligomers of pyrrole derivatives) generated at any step of the synthesis, do not show pseudo-capacitance when analyzed using electrochemical methods, and thus we carried out electrochemical study only for the final products. In proposed synthesis methods, the last phase is a chemical crosslinking of the pyrrole using the ammonium persulfate in aqueous acidic medium as the crosslinking agent. Simultaneously, we have applied other methods for the crosslinking of pyrrole with the methacrylate backbone, which was initially connected to the carbon (not to monomer). Fig. S16 (ESI $\dagger$ ) shows results related to the electrochemical polymerization of the pyrrole onto the methacrylic backbone distributed on the carbon surface. The narrow CV scan indicates on a low gravimetric capacitance. This demonstrates that the method of synthesis resulted in the product that is not electrochemically active. Another drawback of the e-ATRP synthesis is copper contamination. ${ }^{26}$ In the proposed crosslinking of pyrrole in the presence of APS (not electrochemical as shown in Fig. S16†), the hydrochloric acid used for the activation of ammonium persulfate, also facilitated the removal of copper, which was confirmed by elemental mapping performed using the energy dispersive X-ray spectroscopy and the cyclic voltammetry.

The specific capacitance of electrodes constructed from the carbon-grafted-poly(pyrrole) was estimated by integrating area under CV curve (Fig. 7), recorded for freshly prepared electrodes and after the long-term stability test. The charge (in coulombs) was standardized to the mass of active components ( $m$ in $g$ ) at the applied potential scan rate $\left(\nu\right.$ in $\left.\mathrm{V} \mathrm{s}^{-1}\right)$. For the charge integrated in the potential window $\left(E_{2}-E_{1}\right.$ in $\left.\mathrm{V}\right)$, the specific capacitance is calculated according to eqn (2):

$$
C\left(\mathrm{~F} \mathrm{~g}^{-1}\right)=\frac{\int_{E_{1}}^{E_{2}} I(E) \mathrm{d} E}{2 m v\left(E_{2}-E_{1}\right)}
$$

Table 1 demonstrates specific capacitances for all products. In summary, the e-ATRP method delivers composites with higher specific capacitances as compared to the same sample prepared by RAFT. Regarding the influence of carbon type on capacitance, the MWCNT-based materials show the best capacitances regardless of the synthesis method, with the maximum capacitance of $456.8 \mathrm{~F} \mathrm{~g}^{-1}$, which is significantly better as compared to the MWCNT-grafted-PPy with the amidelike linker $\left(350 \mathrm{~F} \mathrm{~g} \mathrm{~g}^{-1}\right),{ }^{16}$ or to bare MWCNTs $\left(90 \mathrm{~F} \mathrm{~g}^{-1}\right.$ (ref. 35) or even lower as demonstrated in this work in Table S1 $\dagger$ ), and to pure PPy (Table S1†). Not only MWCNT-based, but other composites showed higher specific capacitances than their individual components, e.g., $4.07 \mathrm{~F} \mathrm{~g}^{-1}$ for pure PPy (this work, Table S1†), $15.00 \mathrm{~F} \mathrm{~g}^{-1} \mathrm{SWCNH}^{16}$ or $11.60 \mathrm{~F} \mathrm{~g}^{-1}$ for bare graphene. ${ }^{16}$ This result demonstrates the synergy of double-layer and pseudo-capacitance in combined materials. The electronic effects are critical for an efficient charge accumulation and transfer in these composites. Both, the morphology and electronic interactions between components in grafted materials are crucial for improvement in specific gravimetric 
Table 1 The specific capacitance $\left(F \mathrm{~g}^{-1}\right)$, diffusion coefficient $(D)$, cycle stability of products obtained by RAFT and e-ATRP

\begin{tabular}{lcll}
\hline Sample & $\begin{array}{l}\text { Capacitance } \\
\left(\mathrm{F} \mathrm{g}^{-1}\right)\end{array}$ & $\begin{array}{l}\text { Diffusion } \\
\text { coefficient } \\
\left(\mathrm{cm}^{2} \mathrm{~s}^{-1}\right)\end{array}$ & $\begin{array}{l}\text { Electrochemical } \\
\text { stability } \\
\text { (cycle no. })\end{array}$ \\
\hline $\begin{array}{l}\text { MWCNT- } g \text {-PPy } \\
\text { (RAFT) }\end{array}$ & 338.43 & $1.06 \times 10^{-8}$ & 7500 \\
$\begin{array}{l}\text { Graphene- } g \text {-PPy } \\
\text { (RAFT) }\end{array}$ & 231.88 & $2.91 \times 10^{-9}$ & 7500 \\
$\begin{array}{l}\text { SWCNH- } \text {-PPy } \\
\text { (RAFT) }\end{array}$ & 98.54 & $4.89 \times 10^{-10}$ & 5400 \\
$\begin{array}{l}\text { MWCNT- } g \text {-PPy } \\
\text { (e-ATRP) }\end{array}$ & 456.86 & $1.53 \times 10^{-8}$ & 6500 \\
$\begin{array}{l}\text { Graphene- } g \text {-PPy } \\
\text { (e-ATRP) }\end{array}$ & 254.01 & $2.65 \times 10^{-9}$ & 6350 \\
$\begin{array}{l}\text { SWCNH- } g \text {-PPy } \\
\text { (e-ATRP) }\end{array}$ & 113.94 & $5.83 \times 10^{-10}$ & 5500 \\
& & &
\end{tabular}

capacitance. ${ }^{36,37}$ An example is the graphene-based composite shown in Fig. $5 \mathrm{~b}$, where the most outer plane of the carbon is coated with a continuous polymer film. This generates two competitive effects. Firstly, it hinders the access of ions to both sides of graphene planes, which is necessary to achieve the maximum of double layer capacitance for this carbon. On the other hand, the stacking of graphene sheets (up to twelve sheets on average based on TEM imaging) observed after grafting, facilitates the intercalation of ions, similar to the process taking place in graphite anodes. This effect, together with a contribution from the PPy pseudo-capacitance, results in improved gravimetric capacitance that reaches $254 \mathrm{~F} \mathrm{~g}^{-1}$ for e-ATRP and $231 \mathrm{~F} \mathrm{~g}^{-1}$ for RAFT products. The graphene-based composites synthetized via the radical living polymerization shows significantly better capacitances as compared to the same composites made by a combined electrochemical and chemical grafting with the amide-type linker, ${ }^{3,16}$ and as compared to capacitances obtained for pristine carbons or pure PPy (Table S1 $\dagger$ ). Also, the difference in electrochemical characteristics of covalently grafted composites for graphene are related to its structure, where the extended $\pi$-systems of the carbon are not accessible for reactants (inert), due to the structure stabilization via $\pi-\pi^{*}$ stacking. This makes the covalent derivatization of graphene rather challenging. ${ }^{34}$ The benefit of using the living radical polymerization in the process of grafting of the graphene with poly(pyrrole) is that the carbon is primarily derivatized with the reactive moiety that prevents agglomeration of carbon and the monomer.

MWCNT-based capacitors fabricated in this study are the best performing electrodes as compared to the graphene- and SWCNHs-grafted composites. Indeed, their enhanced doublelayer capacitance is related to the MWCNT morphology. The uniform in size and shape long tubes, also named as a onedimensional structures, favor the specific electronic interactions within inner shells and along outer graphene layers. ${ }^{35}$ The interactions between these surfaces create an extended electrical field resulting in improved electrochemical double layer capacitance. ${ }^{14,38}$ The most evident benefit of RAFT and e-ATRP grafting is observed for the SWCNH-based composites. Firstly, the combined materials showed a major improvement of the total capacitance as compared to bare nanohorns $\left(15.00 \mathrm{~F} \mathrm{~g}^{-1}\right.$ (ref. 16) or in Table S1†), and at least two times higher than grafted materials via different organic moieties., ${ }^{3,14}$ This, again refers to the morphology of composites, revealing the benefit of using the living polymerization over other chemical and electrochemical grafting of carbon. As observed in previous work, ${ }^{14}$ the SWCNH-grafted-PPy tents to agglomerate to extent that the double-layer capacitance from the carbon fraction is obstructed. Due to the size of a needle-like carbon ("horns"), in the range of several nanometer (TEM of bare SWCNH are shown in Fig. S10 in ESI $\dagger$ ), the grafting of this nano-structures with other components leads, in most cases, to covering of entire carbon, and therefore to the electrochemical response only from the pseudo-capacitive polymer. Fig. 5d shows the TEM image of SWCNH-grafted-PPy, where very small polymer beads can be recognized onto the carbon together with tiny polymer flakes that appear locally within colonies of carbon "horns". Since carbon is open to the ion from electrolyte and the polymer size facilitate the ion transport within its bulk, these both effects contribute to the improvement of gravimetric capacitance of the material. The pseudo-capacitance depends on the electrode polarization, thus peaks responsible for the poly(pyrrole) activity may be seen in the Fig. 7, inserts A and B. ${ }^{39}$

The ionic mass transport is critical to the effective rate of charge-discharge in capacitor electrodes. The diffusion coefficient $(D)$ is an indication on the ion transport within the material and is analyzed in this work using coulometry technique. The data are further fitted using linear function of charge $(Q)=f($ square root of time, $\sqrt{t}$ ) according to the Cottrell equation (eqn (3)):

$$
Q(\mathrm{C})=2 n F A \sqrt{D \pi} \sqrt{t}
$$

where $D$ is the diffusion coefficient in $\mathrm{cm}^{2} \mathrm{~s}^{-1}, n$ is a number of electrons exchanged in the polymer unit (assumed 1 for polypyrrole), $F$ corresponds to the Faraday constant $96485 \mathrm{C} \mathrm{mol}^{-1}$ $\mathrm{K}^{-1}$ and $A$ is the geometric are of rotating disk electrode $(0.1963$ $\mathrm{cm}^{2}$ ). Fig. 7a shows an example of the charge $=f$ (square root of time) linear dependence, which slope is used to calculated $D$ values for all composites (Table 1). In general, the product with a maximum gravimetric capacitance shows the highest diffusion coefficient, regardless of the synthesis method. More importantly, for the same type of composite (the same carbon used for the grafting) prepared either by RAFT or e-ATRP, the diffusion coefficient are very similar, leading to the conclusions that the morphologies, in particular the thickness (or particle size) of the polymer and overall carbon coverage are similar (also confirmed by analysis of D and $\mathrm{G}$ bands intensities by Raman spectroscopy). Based on calculated $D$ values (Table 1), the conclusion is that MWCNTs is the best carbon in terms of effective mass transport ( $D$ is the highest), followed by the graphene and SWCNHs. MWCNTs-grafted-PPy showed the best gravimetric capacitance in both RAFT and e-ATRP products. Thus, the type of carbon used as grafting core is a key component in these composites. 
The differences in mass transport (diffusion coefficient) further affects ion doping and the rate of redox activity of the polymer. The differential pulse voltammograms (DPV) shown in Fig. $8 \mathrm{~b}-\mathrm{d}$, demonstrate the voltammetry response of the MWCNT-grafted-PPy prepared by e-ATRP (b), graphene-graftedPPy by e-ATRP technique (c), and the SWCNH-grafted-PPy synthesized by RAFT (d). The improved mass transport results in stronger redox signals (higher redox current over doublelayer current). For example, the formation of amide polaron is observed at $0.824 \mathrm{~V}$ for the MWCNT-grafted-PPy prepared by the e-ATRP technique (with the highest diffusion coefficient) and the poly(pyrrole) activity at $0.556 \mathrm{~V}$, at $-0.48 \mathrm{~V}$ and at $-0.204 \mathrm{~V}$. Also, the reduction of the amide bond is seen at $-0.844 \mathrm{~V}$ and $-0.724 \mathrm{~V}$. A weak signal corresponding to the chloride reduction appears at $-0.081 \mathrm{~V}$. The similar transitions were detected for the graphene-grafted-PPy (c) prepared by the e-ATRP technique, with minor shifts towards higher potentials, such as the polaron formation of amide linker accompanied by the second reduction at $-0.792 \mathrm{~V}$ or the poly(pyrrole) activity at $0.528 \mathrm{~V}$ and $-0.264 \mathrm{~V}$. These shifts are attributed to the electronic effects within the graphene and polymer conjugated double-bonds. The corresponding peaks are identified for the SWCNHgrafted-PPy prepared by RAFT (d) at $0.484 \mathrm{~V}$ and $-0.264 \mathrm{~V}$, which are related to the poly(pyrrole) activity, and the reduction
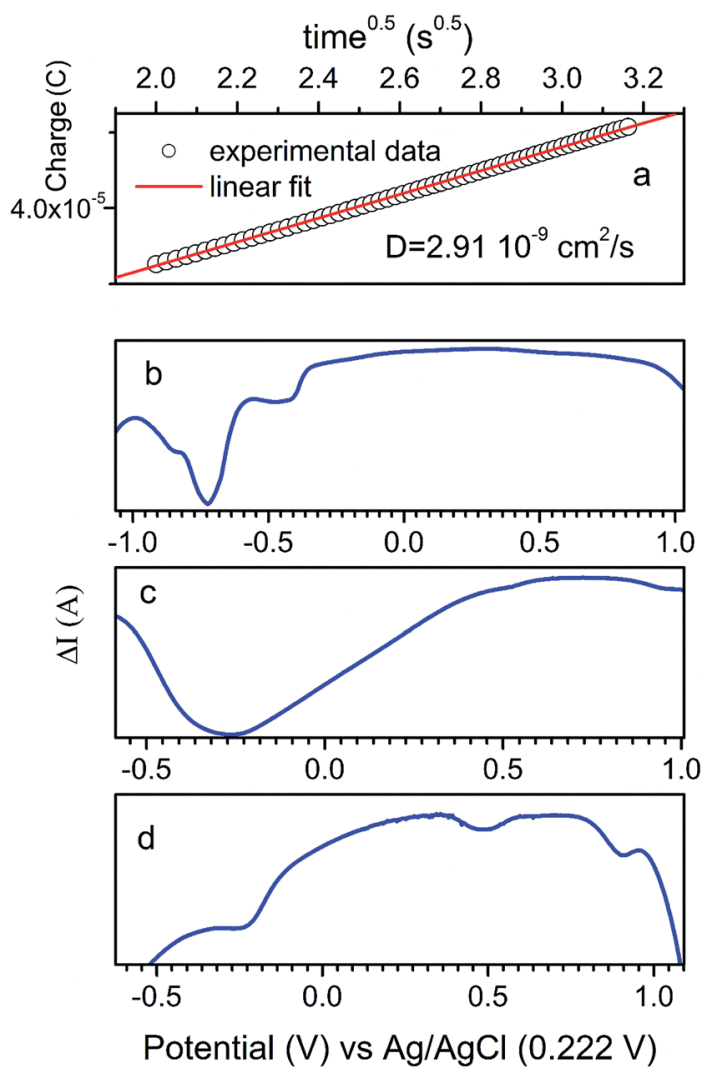

Fig. 8 An exemplary Anson plot for SWCNH-g-PPy prepared by RAFT polymerization used for analysis of diffusion coefficient (a); differential pulse voltammetry scans for MWCNT-g-PPy prepared by e-ATRP polymerization (b), graphene-g-PPy prepared by RAFT (c) and SWCNH-g-PPy prepared by RAFT (d). of dithionic endcapped polymer at $0.908 \mathrm{~V}$. For both, the graphene- and SWCNH-based electrodes, the redox signals are weaker as compared to the MWCNTs-composites, due to their slower mass transport (smaller diffusion coefficients).

Regarding the electrochemical stability investigated by chronopotentiometry (Fig. S14†), materials synthetized by the RAFT showed slightly better stability as compared to systems prepared by e-ATRP. Based on earlier observations, the morphology of composites consisting of the same type of carbon, but prepared using different living polymerization are very similar, and also the thickness (or the particle size) of the polypyrrole are comparable. Thus the stability of materials must be related to chemical structure of the covalent linker. We predict that the chemical stability of the RAFT products are better that those synthesized by the e-ATRP, due to possible crosslinking between more branched polymeric chains for RAFT materials. Also, the oxygen containing functionalities in polyacrylates are not stable under oxidizing potential. ${ }^{40} \mathrm{~A}$ possible solution would be to engage the sulfur end-capped chains, as it was done for e-ATRP. Overall, this chemical modification makes e-ATRP composites still relatively stable, as indicated by multiple chronopotentiometric scans (Fig. S14† and Table 1).

In terms of carbon type, both MWCNT-grafted and graphenegrafted-PPy show better stability as compared to the SWCNHbased materials. This suggest more rigid chemical and electrochemical structures for first two composites, resulting in better stability of the polymer, and is generated by stronger electronic interaction between the PPy and the carbon $\pi$ systems. As observed for the SWCNH-grafted-PPy (Fig. 5d), the polymer is mostly located between the carbon nano-clusters, and because of their very small size, it accumulates within the carbon macropores instead (not on the surface of small "horns"). Presumably, there is less chemical bonding between the PPy and SWCNHs, resulting in luck of covalent rigidness, also less physical contact between these components (means less electronic interaction via $\pi$ stacking), resulting in unstable material (for both RAFT and e-ATRP SWCNHs products). The stability tests showed that the MWCNT-based composite electrodes are more stable as compared to graphene or SWCNHbased peers. In addition, the volumetric changes of poly(pyrrole) during charge-discharge cycles seems to be amplified for the edge-rich morphologies, such as in SWCNH, therefore their mechanical stability (and electrochemical performance) will be deteriorated. In case of graphene, the stability is improved, as the electronic interactions between carbon monolayers and the organic phase are strong. ${ }^{\mathbf{1 4}}$ Likely, this effect also contributes to improved stability of the MWCNTbased composite (multi-walled tubes are made from several stacked layers of graphene). The SWCNHs are self-standing, separated structures made from ultra-small carbon "horns". Therefore, not only the electronic interactions are weaker (it is a single-walled carbon), but the polymer is mostly accumulated within the carbon clusters, therefore its contact with carbon is limited, and it degrades similar to that of pure polypyrrole. The SWCNH-grafted-PPy requires further optimization of the structure, which leads to smaller size of PPy particles. 

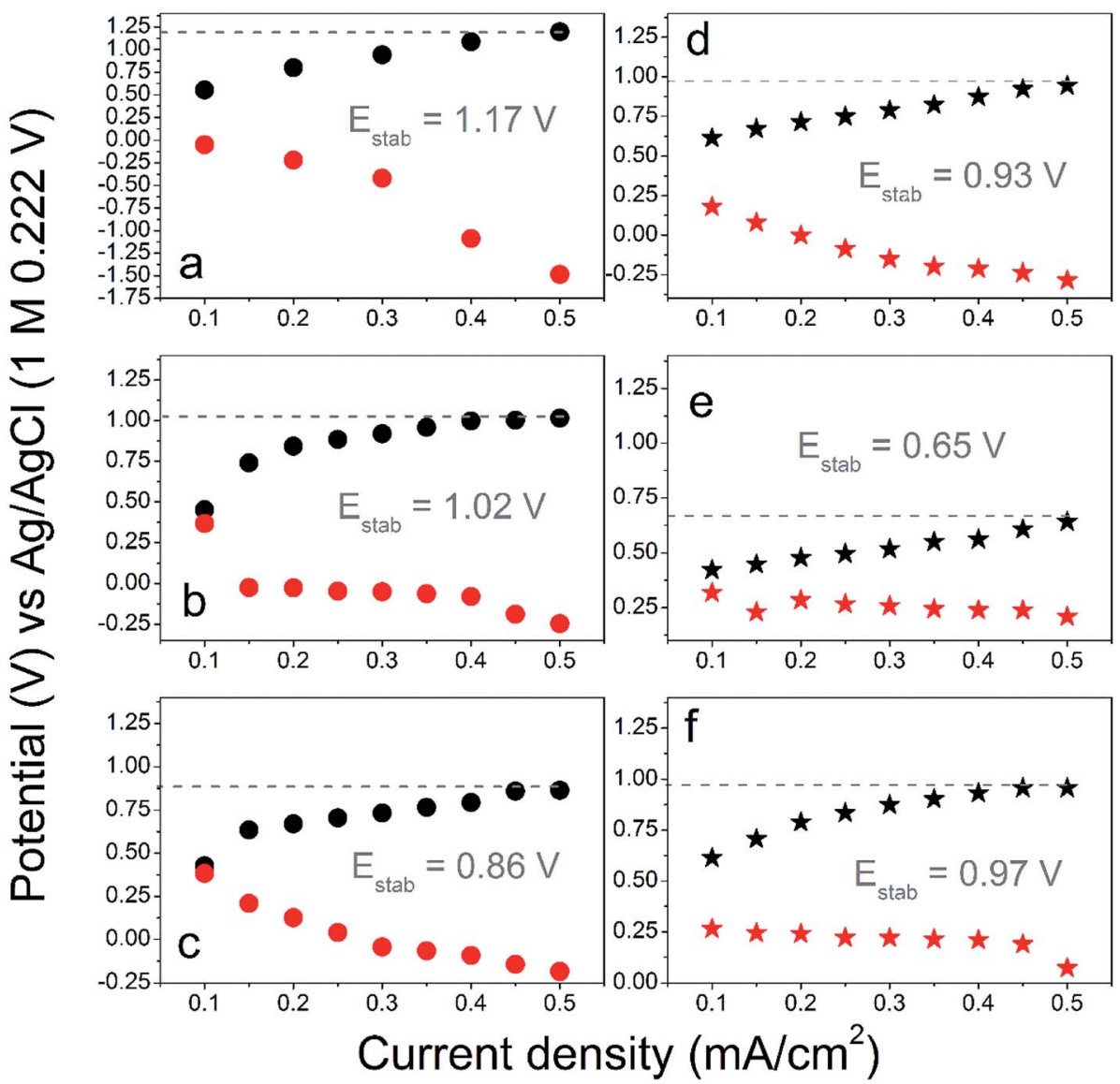

Fig. 9 The charge-discharge potential at various current load for capacitor electrode made form RAFT composites (a-c) and e-ATRP ( $d-f$ ) for MWCNT-g-PPy ( $a$ and d), graphene-g-PPy (b and e) and SWCNH-g-PPy (c and f).

Fig. 9 summarizes the electrode equilibrium voltage as the function of applied current load and was constructed from the long term charge-discharge curves (Fig. S14 $\dagger$ ). Overall, the composites made from grafted MWCNT showed the highest electrode voltage, only slightly smaller is observed for graphenebased composites, and the lowest for SWCNH-grafted PPy (made by RAFT). This correlates well with morphology observations and the analysis of mass transport. The composites prepared by e-ATRP showed slightly lower electrode voltages as compared to products of the RAFT. More importantly, all composites revealed higher electrode voltage as compared to bare polypyrrole (stable at $0.68 \mathrm{~V} v s . \mathrm{Ag} / \mathrm{AgCl}$ ).

This demonstrates that carbon is important stabilizing component in such materials, and emphasizes benefit from the covalent grafting with polymer (only e-ATRP-made graphene showed some discrepancy, presumably due to less porous structure of the e-ATRP product). Both, the graphene- and SWCNH-grafted-PPy can be further optimized in the e-ATRP process, leading to more uniform distribution of the polymer, better separation of graphene sheets by the polymer (for graphene-based), and to decrease in the polymer particle size for the SWCNH system. Since the morphology of both RAFT and e-ATRP products are similar, the voltage stability of electrode is apparently related to the chemical structure and stability of molecular linker. For the RAFT-grafted composites, the chemical linker composed of thiocarbonyl functionalities is more stable under oxidizing potential as compared to the linker used in the e-ATRP. In the latest, the amide and ester motes are degraded faster via bond breaking, due to the formation of a destructive polaron. It causes materials synthesized via the eATRP to be more prone to electrochemical degradation, while the product of RAFT contains more carbon atoms with $\mathrm{sp}^{3}-\mathrm{sp}^{3}$ structure, which are more stable. In total, this results in slight improvement of the cycle-life and higher electrode voltages for materials synthesized by the RAFT method.

\section{Conclusions}

In this study two living radical polymerization techniques, e-ATRP and RAFT, were applied for the grafting carbon allotropes such as MWCNT, graphene and SWCNH with the methacrylate functionalized-poly(pyrrole). The modified carbons were examined as the polymerization initiators in the e-ATRP and RAFT synthesis. Their electrochemical response for the co-catalyst activity revealed the highest activation rate constant for SWCNH, owing to its highest specific surface area, and thus availability of large electrochemical interface for the redox process. The Fourier-transform infrared and Raman spectroscopies were used to identify the 
reaction products at each phase and for the final composites. TEM imaging showed that: (i) the morphology of composites made from the same carbon allotrope are not significantly different for RAFT and e-ATRP products; (ii) the structure of the ultimate product strongly depends on the type of carbon: for SWCNH the polymer was locally agglomerated between the carbon clusters due to ultrasmall carbon particles, and for the graphene, the polymer formed an uniform layer on the most outer sheets of graphene; (iii) the poly(pyrrole) film or the particle size was very small (in all cases less than $30 \mathrm{~nm}$ ), demonstrating the control over the polymer morphology in living polymerization techniques. The high specific gravimetric capacitances over $456 \mathrm{~F} \mathrm{~g} \mathrm{~g}^{-1}$ and electrochemical stability up to 7500 cycles were obtained for MWCNT-graftedpoly(pyrrole), and slightly less for graphene-based composites synthetized by e-ATRP, showing the advantages of this method over RAFT. The electrode voltages for all composites were higher as compared to the pure polymer electrodes, with some benefit of RAFT over e-ATRP product, and with significant improvement observed for the MWCNT- and graphene-based systems. Regardless of the synthesis method, all composites demonstrated enhanced specific capacitance as compared to their individual components, revealing the synergy of double-layer capacitance from the carbon and the pseudo-capacitance generated by the polymer fraction. Both, the RAFT and e-ATRP are polymerization methods that deliver nanomaterials with tailored morphology and desired functions, by creating a stable anchorage between carbon, molecular linker and the polymer, and without unwanted side effects such as the homopolymerization, as indicated in our previous studies on similar carbon/poly(pyrrole) systems. ${ }^{\mathbf{1 6}}$

\section{Acknowledgements}

This work was carried out with the financial support of New Brunswick Foundation for Innovation, Frank J. and Norah Toole Graduate Scholarship and UNB President.

\section{References}

1 D. Andre, S.-J. Kim, P. Lamp, S. F. Lux, F. Maglia, O. Paschosa and B. Stiaszny, Future Generations of Cathode Materials: An Automotive Industry Perspective, J. Mater. Chem. A, 2015, 3, 6709-6732.

2 A. González, E. Goikolea, J. A. Barrena and R. Mysyk, Review on Supercapacitors: Technologies and Materials, Renewable Sustainable Energy Rev., 2016, 58, 1189-1206.

3 M. Radtke and A. Ignaszak, A Surface Grafting of Carbon Allotropes with in situ Generated 3-aryl Diazonium Chlorides: Electrochemical Kinetic Studies, Electroanalysis, 2016, 28, 2900-2909.

4 Y. Liu and X. Peng, Recent Advances of Supercapacitors Based on Two-Dimensional Materials, Applied Materials Today, 2017, 7, 1-12.

5 T. Chen and L. Dai, Carbon Nanomaterials for High Performance Supercapacitors, Mater. Today, 2013, 16, 272280.

6 T. Qian, X. Zhou, C. Yu, S. Wu and J. Shen, Highly Dispersed Carbon Nanotube/Polypyrrole Core/Shell Composites with
Improved Electrochemical Capacitive Performance, $J$. Mater. Chem. A, 2013, 1, 15230-15234.

7 A. M. Abioye and F. N. Ani, Recent Development in the Production of Activated Carbon Electrodes from Agricultural Waste Biomass for Supercapacitors: A review, Renewable Sustainable Energy Rev., 2015, 52, 1282-1293.

8 Z. Zondaka, R. Valner, T. Tamm, A. Aabloo and R. Kiefer, Carbide-Derived Carbon in Polypyrrole Changing the Elastic Modulus with a Huge Impact on Actuation, RSC Adv., 2016, 6, 26380-26385.

9 N. Maheswari and G. Muralidharan, Supercapacitor Behavior of Cerium Oxide Nanoparticles in Neutral Aqueous Electrolytes, Energy Fuels, 2015, 29, 8246-8253.

10 H. Ji, X. Zhao, Z. Qiao, J. Jung, Y. Zhu, Y. Lu, L. Zhang, A. H. MacDonald and R. S. Ruoff, Capacitance of CarbonBased Electrical Double-Layer Capacitors, Nat. Commun., 2014, 5, 1-7.

11 A. M. Bryan, L. M. Santino, Y. Lu, S. Acharya and J. M. D'Arcy, Conducting Polymers for Pseudocapacitive Energy Storage, Chem. Mater., 2016, 28, 5989-5998.

12 L. L. Zhang, R. Zhou and X. S. Zhao, Carbon-Based Materials as Supercapacitor Electrodes, J. Mater. Chem., 2009, 38, 2520-2531.

13 U. Patil, S. C. Lee, S. Kulkarni, J. S. Sohn, M. S. Nam, S. Han and S. C. Jun, Nanostructured Pseudocapacitive Materials Decorated 3D Graphene Foam Electrodes for Next Generation Supercapacitors, Nanoscale, 2015, 7, 6999-7021. 14 M. Radtke, D. G. G. McMillan, B. Schroter, S. Hoppener, B. Dietzek, U. S. Schubert and A. Ignaszak, The Effect of 3amino Benzoic Acid Linker and the Reversal of DonorAcceptor Pairs on the Electrochemical Performance and Stability of Covalently Bonded Poly(pyrrole) Nanotubes, Polymer, 2015, 77, 289-296.

15 K. Shi and I. Zhitomirsky, Fabrication of Polypyrrole-Coated Carbon Nanotubes Using Oxidant-Surfactant Nanocrystals for Supercapacitor Electrodes with High Mass Loading and Enhanced Performance, ACS Appl. Mater. Interfaces, 2013, 5, 13161-13170.

16 M. Radtke and A. Ignaszak, Grafting of the carbon Allotropes and Polypyrrole via a Kevlar-Type Organic Linker: The Correlation of Carbon Structure/Morphology with Electrochemistry of the Composite Electrode, Mater. Renew. Sustain. Energy, 2017, 6, 1-15.

17 C. Cioffi, S. Campidelli, C. Sooambar, M. Marcaccio, G. Marcolongo, M. Meneghetti, D. Paolucci, F. Paolucci, C. Ehli, G. M. A. Rahman, V. Sgobba, D. M. Guldi and M. Prato, Synthesis, Characterization, and Photoinduced Electron Transfer in Functionalized Single Wall Carbon Nanohorns, J. Am. Chem. Soc., 2007, 129, 3938-3945.

18 S. Mahouche-Chergui, S. Gam-Derouich, C. Mangeney and M. M. Chehimi, Aryl Diazonium Salts: A New Class of Coupling Agents for Bonding Polymers, Biomacromolecules and Nanoparticles to Surfaces, Chem. Soc. Rev., 2011, 40, 4143-4166.

19 Z. Huang, L. Zhang, Z. Cheng and X. Zhu, Reversible Addition-Fragmentation Chain Transfer Polymerization of 
Acrylonitrile under Irradiation of Blue LED Light, Polymer, 2017, 9, 4-8.

20 S. S. Rane and P. Choi, Polydispersity Index: How Accurately Does It Measure the Breadth of the Molecular Weight Distribution?, Chem. Mater., 2005, 17, 926.

21 J. Ma and R. M. Larsen, Comparative Study on Dispersion and Interfacial Properties of Single Walled Carbon Nanotube/Polymer Composites Using Hansen Solubility Parameters, ACS Appl. Mater. Interfaces, 2013, 5, 1287-1293.

22 K. Matyjaszewski and J. Spanswick, Controlled/Living Radical Polymerization, Mater. Today, 2005, 8, 26-33.

23 K. Matyjaszewski and N. V. Tsarevsky, Nanostructured Functional Materials Prepared by Atom Transfer Radical Polymerization, Nat. Chem., 2009, 1, 276-288.

24 K. T. Constantopoulos, C. J. Shearer, J. G. Shapter, N. H. Voelcker and A. V. Ellis, Preparation and Characterization of Multiwalled Carbon Nanotube (MWCNT)/Polymer Nanostructured Materials, Proc. SPIE, 2008, 7267, 72670G.

25 V. Eswaraiah, V. Sankaranarayanan and S. Ramaprabhu, Inorganic Nanotubes Reinforced Polyvinylidene Fluoride Composites as Low-Cost Electromagnetic Interference Shielding Materials, Nanoscale Res. Lett., 2011, 6, 1-11.

26 M. Nasser-Eddine, C. Delaite, P. Dumas, R. Vataj and A. Louati, Copper Removal in Atom Transfer Radical Polymerization Through Electrodeposition, Macromol. Mater. Eng., 2004, 289, 204-207.

27 S. S. Hosseiny and P. van Rijn, Surface Initiated Polymerizations via e-ATRP in Pure Water, Polymers, 2013, 5, 1229-1240.

28 A. J. D. Magenau, N. C. Strandwitz, A. Gennaro and K. Matyjaszewski, Electrochemically Mediated Atom Transfer Radical Polymerization, Science, 2011, 332, 81-84.

29 X. An, X. He, L. Chen and Y. Zhang, Graphene Oxide-Based Boronate Polymer Brushes via Surface Initiated Atom Transfer Radical Polymerization for the Selective Enrichment of Glycoproteins, J. Mater. Chem. B, 2016, 4, 6125-6133.
$30 \mathrm{~W}$. Tang and K. Matyjaszewski, Effect of Ligand Structure on Activation Rate Constants in ATRP, Macromolecules, 2006, 39, 4953-4959.

31 C. J. Pouchert, The Aldrich library of FT-IR spectra, Aldrich Chemical Company, Wis.: Aldrich, Milwaukee, 1997, vol. 3.

32 T. Liu, T. Liu, L. Finn, M. Yu, H. Wang, T. Zhai, X. Lu, Y. Tong and Y. Li, Polyaniline and Polypyrrole Pseudocapacitor Electrodes with Excellent Cycling Stability, Nano Lett., 2014, 14, 2522-2527.

33 J. J. Miasik, A. Hooper and B. C. Tofield, Conducting Polymer Gas Sensors, J. Chem. Soc., Faraday Trans. 1, 1986, 82, 17.

34 Macromolecular Systems - Materials Approach, ed. Y. Osada and D. E. De Rossi, Springer-Verlag, Berlin, 2000.

35 H. Pan, J. Li and Y. P. Feng, Carbon Nanotubes for Supercapacitor, Nanoscale Res. Lett., 2010, 5, 654-668.

36 S. Devaramani, P. S. Adarakatti and P. Malingappa, Covalent Anchoring of Cobalt Hexacyanoferrate Particles on Graphitic Carbon: A Simple and Renewable Robust Pellet Electrode as an Electrochemical Interface for Amperometric Quantification of Sulfite, Electrochim. Acta, 2017, 231, 650658.

37 M. Cuartero, J. Bishop, R. Walker, R. G. Acres, E. Bakker, R. De Marcobde and G. Crespo, Evidence of Double Layer/ Capacitive Charging in Carbon Nanomaterial-based Solid Contact Polymeric Ion-Selective Electrodes, Chem. Commun., 2016, 52, 9703-9706.

38 K. Ketpang and J. S. Park, Electrospinning PVDF/PPy/ MWCNTs Conducting Composites, Synth. Met., 2010, 160, 1603-1608.

39 B. E. Conway and E. Gileadi, Kinetic theory of pseudocapacitance and electrode reactions at appreciable surface coverage, Trans. Faraday Soc., 1962, 58, 2493-2509.

40 B. Häupler, A. Ignaszak, T. Janoschka, T. Jähnert, M. D. Hager and U. S. Schubert, Poly(methacrylates) with Pendant Benzoquinone Units - Monomer Synthesis, Polymerization, and Electrochemical behavior: Potential New Polymer Systems for Organic Batteries, Macromol. Chem. Phys., 2014, 215, 1250-1256. 